\title{
Multiplier Theory for Stability Analysis of Anti-Windup Control Systems*
}

\author{
Mayuresh V. Kothare \\ †Chemical Engineering 210-41 \\ California Institute of Technology \\ Pasadena, CA 91125, U.S.A.
}

\author{
Manfred Morari ${ }^{1} \ddagger$ \\ $\ddagger$ Automatic Control Laboratory \\ Swiss Federal Institute of Technology (ETH) \\ Physikstrasse 3, 8092 Zürich, Switzerland
}

\section{CDS Technical Report No. CIT/CDS 96-012}

\author{
August 8, 1996
}

Key Words: Control input nonlinearity, constrained system, nonlinear system, stability analysis, anti-windup, bumpless transfer, passivity theorem, linear matrix inequality, multiplier theory, absolute stability.

\begin{abstract}
We apply the passivity theorem with appropriate choice of multipliers to develop sufficient conditions for stability of the general anti-windup bumpless transfer (AWBT) framework presented in [24]. For appropriate choices of the multipliers, we show that these tests can be performed using convex optimization over linear matrix inequalities (LMIs). We show that a number of previously reported attempts to analyze stability of AWBT control systems, using such well-known and seemingly diverse techniques as the Popov, Circle and Off-Axis Circle criteria, the optimally scaled small-gain theorem (generalized $\mu$ upper bound) and describing functions, are all special cases of the general conditions developed in this paper. The sufficient conditions are complemented by necessary conditions for internal stability of the AWBT compensated system. Using an example, we show how these tests can be used to analyze the stability properties of a typical anti-windup control scheme.
\end{abstract}

\section{Introduction}

All real world control systems must deal with constraints. Of special interest are systems with control input constraints in an otherwise linear system. The most common example of an input constraint is actuator saturation. Typically, a valve controlling the flow rate of

*Submitted to Automatica. Also presented at the $34^{\text {th }}$ IEEE Conference on Decision and Control, New Orleans, LO, December 1995.

${ }^{1}$ Corresponding author: phone +411632 7626, fax +411632 1211, e-mail morari@aut.ee.ethz.ch 
the coolant to a reactor can only operate between fully open and fully closed. We will refer to such a constraint as a plant input limitation. In addition, to satisfy multiple objectives, commonly encountered control schemes use overrides or selectors which switch between a "bank" of linear controllers, each of which is designed to achieve a specific objective. We will refer to such a mode switch as a plant input substitution. As a result of substitutions and limitations, the actual plant input will be different from the output of the controller. Thus the controller output does not drive the plant and hence the states of the controller are wrongly updated. This effect is called controller windup.

As is commonly the case, if the linear controller is designed ignoring these actuator nonlinearities, the adverse effect of controller windup is in the form of significant performance degradation, large overshoots in the output and sometimes even instability. In addition, during controller mode switches, the difference between the outputs of different controllers results in a bump discontinuity in the plant input. This, in turn, causes undesirable bumps in the controlled variables. What is required is a smooth transition or bumpless transfer between the different operating modes.

The problem of control system analysis and controller synthesis for the general class of linear time-invariant (LTI) systems subject to plant input limitations and substitutions is referred to as the anti-windup bumpless transfer (AWBT) problem. All known LTI AWBT schemes adopt the following two-step design paradigm:

Design first the linear controller ignoring control input nonlinearities and then add AWBT compensation to minimize the adverse effects of any control input nonlinearities on closedloop performance.

Optimal control strategies, such as model predictive control (MPC), which explicitly account for both input and output constraints are well-known, but computationally expensive. However, AWBT compensation schemes of the type described above provide a simpler, computationally cheaper alternative for modifying or "retro-fitting" existing unconstrained controllers to account for input nonlinearities, particularly in systems where the effect of control input constraints is not expected to be critical. This has been the motivation for a number of AWBT schemes which have been reported in the literature, dating back to the early work of Fertik and Ross (1967) [16] on anti-reset windup. Most of these schemes are somewhat ad-hoc and tailored to specific problems based on spurts of engineering ingenuity. Recently, Kothare et al. (1994) [24] presented a general AWBT framework based on the twostep design concept mentioned above, and, for the first time, established a firm theoretical basis for AWBT control. The resulting AWBT scheme in [24] was shown to unify all known LTI AWBT schemes in terms of two matrix parameters. This significantly clarified the basic underlying concept of AWBT and simplified the problem of comparing and contrasting various previously reported heuristically based AWBT methodologies.

A necessary step in the further development of a complete AWBT theory is the development of tools for analyzing stability of AWBT control systems with or without plant model uncertainty. Below, we summarize the existing literature in this area:

- Glattfelder et al. $[19,20,21]$ analyzed the stability of single input single output (SISO) anti-reset windup PI controllers using the Popov and Circle criteria.

- Kapasouris and Athans (1985) [22] applied a multivariable version of the Circle Crite- 
rion to analyze stability of their multivariable nonlinear anti-reset windup scheme.

- Zheng et al. (1994) [34] used the Off-axis Circle Criterion to establish stability of their anti-windup scheme for internal model control (IMC).

- Åström and Rundqwist (1989) [2] suggested the use of describing function theory to analyze stability of the observer-based anti-windup scheme.

- Doyle et al. (1987) [15] analyzed the stability of their modified anti-windup (MAW) scheme by using extensions of $\mu$-analysis for LTI systems with structured uncertainty to nonlinear systems [14]. A similar analysis was presented by Campo et al. [8, 9].

From the preceding review, we see that several seemingly diverse techniques have been applied to develop stability conditions for several specific AWBT schemes. Very little work has been done on AWBT stability analysis in a reasonably general setting. The objective of this paper is to develop general tools for analyzing the stability properties of the AWBT framework presented in [24]. Since the framework in [24] unifies essentially all known LTI AWBT schemes, the results we obtain are general enough to be applicable to all existing AWBT schemes. A second objective is to show that the technique we use to analyze AWBT stability allows us to interpret and generalize previously reported AWBT stability results in a single unified setting.

The paper is organized as follows: In $\S 2$, we review the general AWBT framework from [24] which is central to the analysis problem under consideration. We also summarize necessary technical machinery such as the absolute stability problem, passivity theorem, multiplier theory and linear matrix inequalities (LMIs), which will be used in the later sections. In $\S 3$, we present the main results on stability of AWBT control systems, under various restrictions on the input nonlinearity. We show how the sufficient conditions for AWBT stability can be checked via readily computable convex conditions involving the feasibility of equivalent LMIs. We also develop necessary conditions for closed-loop stability of the AWBT system. In $\S 4$, we present an example to illustrate the application of the stability results to a typical AWBT control scheme. Finally, in $\S 5$, we present conclusions.

\section{Notation}

The notation is fairly standard. $\Re$ is the set of real numbers. For a matrix $A, A^{T}$ denotes its transpose, $A^{*}$ denotes its complex conjugate transpose, $A^{-1}$ denotes its inverse (if it exists), $A^{-*}$ denotes the inverse of $A^{*}$ (if it exists). The matrix inequality $A>B(A \geq B)$ means that $A$ and $B$ are square Hermitian matrices and $A-B$ is positive (semi-)definite. $\mathcal{L}_{2}$ is the Hilbert space of $m$-vector valued signals defined on $(-\infty, \infty)$, with scalar product $\langle x \mid y\rangle=\int_{-\infty}^{\infty} x(t)^{*} y(t) d t$ and such that $\|x\|_{2} \triangleq\langle x \mid x\rangle^{\frac{1}{2}}<\infty \forall x \in \mathcal{L}_{2} . \mathcal{L}_{2 e}$ is the extended Hilbert space of $m$-vector valued signals $u$ such that $u_{T} \in \mathcal{L}_{2}$, where

$$
u_{T}(t)= \begin{cases}u(t) & \text { if } t \leq T \\ 0 & \text { if } t>T\end{cases}
$$


A transfer function matrix in terms of state-space data is denoted $G(s)=C(s I-A)^{-1} B+D \triangleq$ $\left[\begin{array}{l|l}A & B \\ \hline C & D\end{array}\right]$. For simplicity of notation, $f \circ G(s) x$ refers to the operation of convolving the impulse response of $G(s)$ with $x$ and then applying the operator $f$. Similar interpretation can be given to $G(s) \circ f x$. With some abuse of notation, we will denote the adjoint of an LTI operator $G(s)$ by $G(-s)^{T}$. Thus, with the usual rules of an adjoint operator

$$
\langle x \mid G(s) y\rangle=\left\langle G(-s)^{T} x \mid y\right\rangle
$$

If $G(s)$ is causal, stable, then its adjoint is considered to be anti-causal, stable.

\section{Background}

\subsection{A General AWBT Framework}

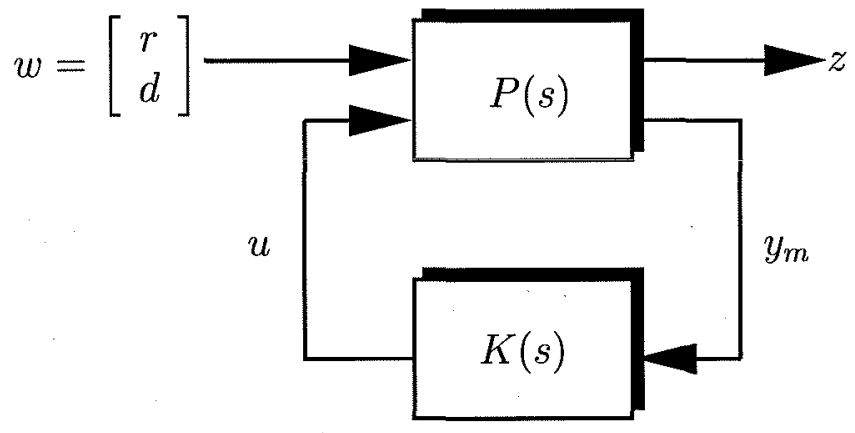

(a) without nonlinearity $N$ (ideal case)

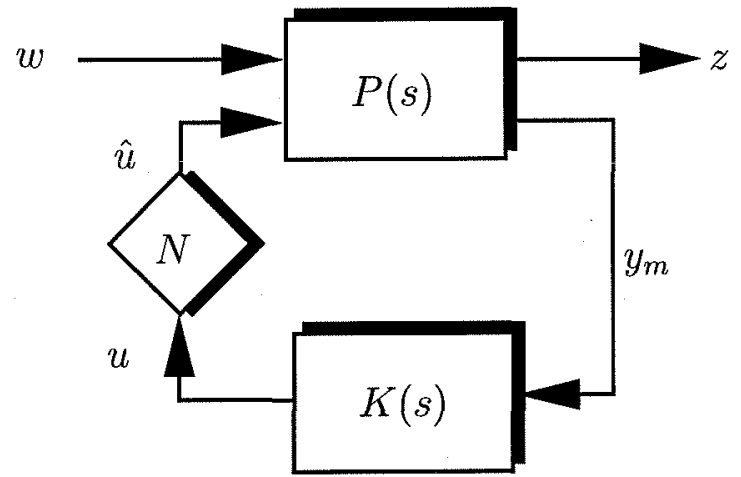

(b) with nonlinearity $N$

Figure 1: Linear control problem

Consider the linear fractional transformation (LFT) in Figure 1(a), which represents the standard linear control problem. $P(s)$ is the LTI plant and $K(s)$ is a stabilizing LTI controller, designed to meet given performance specifications (for example, $\mathcal{H}_{2}$ or $\mathcal{H}_{\infty}$ performance criteria [13]). The exogenous input $w$ includes all signals which enter the system such as commands, disturbances and sensor noise. The input $u$ is the control effort applied to the plant by the controller $K(s)$. The plant outputs $z$ and $y_{m}$ represent the controlled output which the controller is designed to keep small (e.g. tracking error) and all measurements available to the controller respectively.

As discussed in $\S 1$, due to limitations and/or substitutions, a nonlinearity $N$ is introduced into the interconnection as shown in Figure 1(b). As a result, the actual plant input $\hat{u}$ will, in general, not be equal to the controller output $u$. This mismatch is the cause for controller windup, controller state initialization errors and a significant transient which must decay after the system returns to the linear regime. This is also the cause for degradation of performance and sometimes instability. 


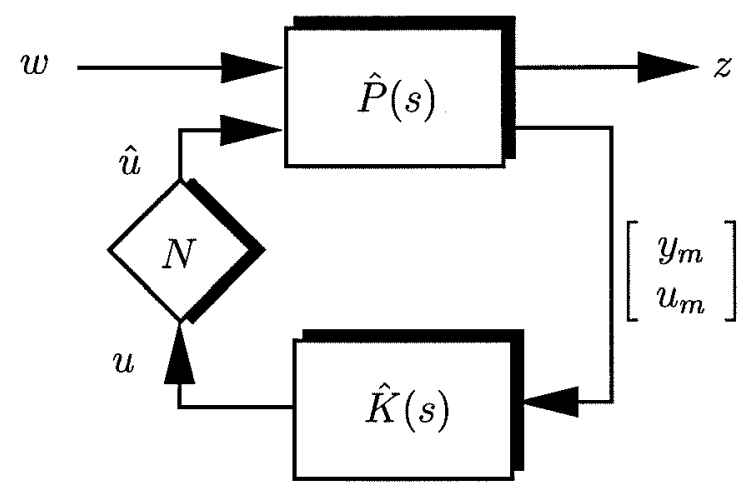

Figure 2: The AWBT problem

The AWBT problem (see Figure 2) involves the design of $\hat{K}(s)$, commonly referred to as the "AWBT compensated version" of $K(s)$. The plant

$$
P(s)=\left[\begin{array}{ll}
P_{11}(s) & P_{12}(s) \\
P_{21}(s) & P_{22}(s)
\end{array}\right]
$$

from Figure 1(b), partitioned according to its inputs and outputs, is augmented to

$$
\hat{P}(s)=\left[\begin{array}{cc}
P_{11}(s) & P_{12}(s) \\
P_{21}(s) & P_{22}(s) \\
0 & P_{32}(s)
\end{array}\right]
$$

in Figure 2, to provide a measurement or estimate

$$
u_{m}=P_{32}(s) \hat{u}
$$

of the plant input $\hat{u}$. We allow the general relationship (3) to account for non-trivial measurement dynamics $\left(P_{32}(s) \not \equiv I\right)$. The measured or estimated value of $\hat{u}$, i.e., $u_{m}$, provides information regarding the effect of the nonlinearity $N$ and is fed back to the AWBT compensated controller $\hat{K}(s)$. The general AWBT problem can be stated as follows:

Given the linear controller $K(s)$ which meets certain linear performance specifications, synthesize the AWBT controller $\hat{K}(s)$ which

- renders the system in Figure 2 stable;

- meets the linear performance specifications when $N \equiv I$; and

- exhibits graceful performance degradation when $N \not \equiv I$.

A parameterization of all AWBT controllers $\hat{K}(s)$ which satisfy certain (appropriately defined) admissibility criteria has been presented in [24]. This parameterization is in terms of two matrix parameters $H_{1}$ and $H_{2}$ and is summarized below:

$$
\text { If } \quad K(s)=V(s)^{-1} U(s)=\left[\begin{array}{l|l}
A & B \\
\hline C & D
\end{array}\right]
$$




$$
\begin{aligned}
& \text { then, } \hat{K}(s)=\left[\begin{array}{ll}
U(s) & I-V(s)
\end{array}\right] \\
& =\left[\begin{array}{c|cc}
A-H_{1} C & B-H_{1} D & H_{1} \\
\hline H_{2} C & H_{2} D & I-H_{2}
\end{array}\right] \\
& \text { where, } \quad U(s)=\left[\begin{array}{c|c}
A-H_{1} C & B-H_{1} D \\
\hline H_{2} C & H_{2} D
\end{array}\right] \\
& V(s)=\left[\begin{array}{c|c}
A-H_{1} C & -H_{1} \\
\hline H_{2} C & H_{2}
\end{array}\right]
\end{aligned}
$$

with $H_{2}$ invertible. Assuming that $H_{1}$ is chosen such that the eigenvalues of $A-H_{1} C$ are in the open left half complex plane, then $U(s)$ and $V(s)$ correspond to the stable left coprime factors of $K(s)$.

The noteworthy feature about this parameterization is that it allows us to unify all known LTI AWBT control schemes under a general framework. Thus, as shown in [24], for particular choices of $H_{1}$ and $H_{2}$, all known LTI AWBT control schemes reported in the literature are special cases of this parameterization, as summarized in Table 1 . The focus of our attention is on analyzing the stability properties of the interconnection in Figure 2.

\begin{tabular}{||l|l|l||}
\hline \hline Parameters & $H_{1}$ & $H_{2}$ \\
\hline \hline Anti-reset windup & $\frac{1}{\tau_{r}}$ & 1 \\
\hline Hanus conditioned controller & $B D^{-1}$ & $I$ \\
\hline Observer-based anti-windup & $L$ & $I$ \\
\hline Conventional anti-windup & $\alpha B(I+\alpha D)^{-1}$ & $(I+\alpha D)^{-1}$ \\
\hline Internal Model Control (IMC) & {$\left[\begin{array}{lll}0 & B_{p}^{T}\end{array}\right]^{T}$} & $I$ \\
\hline Anti-windup IMC & {$\left[\begin{array}{lll}0 & B_{2}^{T} & B_{p}^{T}\end{array}\right]^{T}$} & $I$ \\
\hline Extended Kalman filter & $B_{p}$ & $I$ \\
\hline Generalized Conditioning-I & $B(D+\rho I)^{-1}$ & $I$ \\
\hline Generalized Conditioning-II & {$\left[\begin{array}{lll}0 & D_{f}^{-T} B_{f}^{T}\end{array}\right]^{T}$} & $I$ \\
\hline \hline
\end{tabular}

Table 1: Special cases of the general framework (see Kothare et al. (1994) for details)

\subsection{The Passivity Theorem and Multiplier Theory}

The approach we adopt in this paper for AWBT stability analysis is based on concepts derived from absolute stability theory (see [12, §VI],[23]). Specifically, we apply the passivity theorem $[11,29,30,31]$ with appropriate choice of multipliers $[4,5,33]$ to develop sufficient conditions for AWBT stability. We begin by giving a formal definition of stability.

Definition 1 (Stability) A causal operator $h: \mathcal{L}_{2 e} \longrightarrow \mathcal{L}_{2 e}$ is $\mathcal{L}_{2}$ stable if $x \in \mathcal{L}_{2} \Rightarrow h x \in$ $\mathcal{L}_{2}$. Furthermore, if $\exists \gamma \geq 0$ and $\beta$ such that

$$
\|h x\|_{2} \leq \gamma\|x\|_{2}+\beta, \quad \forall x \in \mathcal{L}_{2}
$$

then, $h$ is said to be finite-gain $\mathcal{L}_{2}$ stable. 
Note that stability requires the output $h x$ to belong to the non-extended space $\mathcal{L}_{2}$ for all $x \in \mathcal{L}_{2}$. A feedback interconnection of the form shown in Figure 3 is $\mathcal{L}_{2}$ stable if all closedloop maps from all external inputs to all internal variables are $\mathcal{L}_{2}$ stable. Finite-gain $\mathcal{L}_{2}$ stability of the interconnection can be defined similarly. Next we define the concept of passivity.

Definition 2 (Passivity) [12] An operator $h: \mathcal{L}_{2 e} \longrightarrow \mathcal{L}_{2 e}$ is said to be strictly passive if $\exists \delta>0$ and some $\beta$ such that

$$
\left\langle x_{T} \mid(h x)_{T}\right\rangle \geq \delta\left\|x_{T}\right\|_{2}^{2}+\beta, \quad \forall T \in \Re, \forall x \in \mathcal{L}_{2 e} .
$$

If $\delta \geq 0$, then $h$ is said to be passive.

The motivation for this definition of passivity comes from network theory where circuit elements which absorb energy are called passive elements. For example, the energy absorbed by a resistance $R>0$ with a voltage $v$ across it and a current $i$ through it is given by $\left\langle v_{T} \mid i_{T}\right\rangle=\int_{0}^{T} i(t) v(t) d t=R \int_{0}^{T} i^{2}(t) d t$ and hence a resistance is a strictly passive element.

If $h$ is a causal, stable and LTI operator with transfer function $H(s)$, then it is (strictly) passive, i.e., it satisfies (9) if and only if there exists $\delta \geq(>) 0$ such that $[12, \S \mathrm{VI}]$

$$
H(j \omega)+H^{*}(j \omega) \geq 2 \delta I, \quad \forall \omega \in \Re .
$$

A matrix transfer function $H(s)$, whether stable or unstable but having no poles on the $j \omega$ axis, and satisfying (10) is said to be generalized (strictly) positive real [1]. The following lemma gives an equivalent condition for checking (10) in terms of the state-space matrices of $H(s)$.

Lemma 1 (Positive Real Lemma) [1] A matrix transfer function $H(s)$ having no poles on the $j \omega$ axis, with a controllable state-space realization $(A, B, C, D)$, satisfies (10) iff there exists a symmetric matrix $Q=Q^{T}$ such that

$$
\left[\begin{array}{cc}
Q A^{T}+A Q & B-Q C^{T} \\
B^{T}-C Q & 2 \delta I-\left(D+D^{T}\right)
\end{array}\right] \leq 0
$$

or equivalently, iff there exists a symmetric matrix $Q=Q^{T}$ such that

$$
\left[\begin{array}{cc}
A^{T} Q+Q A & Q B-C^{T} \\
B^{T} Q-C & 2 \delta I-\left(D+D^{T}\right)
\end{array}\right] \leq 0 .
$$

Remark 1 If $H(s)$ is stable, then the matrix $Q=Q^{T}$ in (11), (12) can be taken to be positive definite without loss of generality.

Note that (11),(12) are matrix inequalities that are affine in $Q$ and $\delta$ and are referred to as Linear Matrix Inequalities (LMIs) in $Q$ and $\delta$ (see [6] for details on LMIs). The significance of reducing a problem to the feasibility of an LMI is that LMI-based problems are convex and can be solved in polynomial-time, i.e., with low computational complexity. There exist powerful and effective algorithms which efficiently compute a feasible solution (if it exists) to 
an LMI problem or verify that none exists [18]. Hence, reducing a problem to the feasibility of an LMI is equivalent to solving that problem.

The connection between passivity and stability of the closed-loop shown in Figure 3 was originally addressed by Sandberg [29] and later by Zames [30, 31]. The basic question that needs to be answered in this context is the following: Is a network consisting of passive elements necessarily stable? We state below a general version of the passivity theorem which answers this question.

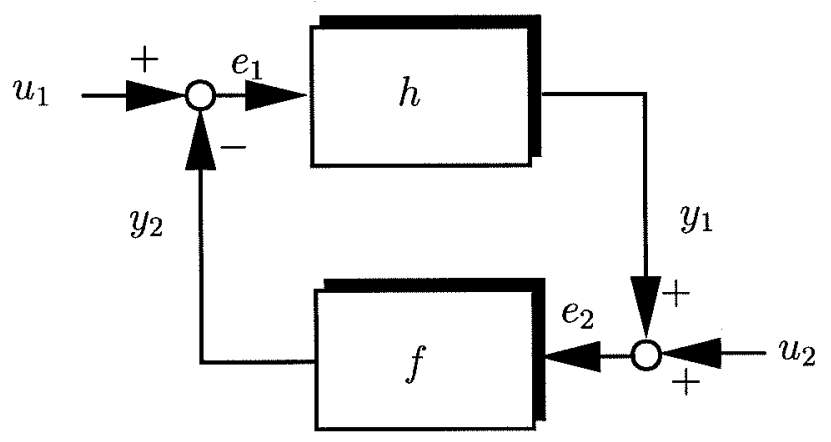

Figure 3: General interconnection for the passivity theorem

Theorem 1 (Passivity Theorem) Consider the feedback system shown in Figure 3, where the operators $h: \mathcal{L}_{2 e} \longrightarrow \mathcal{L}_{2 e}$ and $f: \mathcal{L}_{2 e} \longrightarrow \mathcal{L}_{2 e}$ are any (possibly nonlinear) causal operators. Assume that for any $u_{1}, u_{2} \in \mathcal{L}_{2}$, there exist solutions $e_{1}, e_{2}, y_{1}, y_{2} \in \mathcal{L}_{2 e}$. Suppose there exist constants $\gamma_{1}, \delta_{1}, \delta_{2}, \alpha_{1}, \beta_{1}, \beta_{2}$ such that $\forall x \in \mathcal{L}_{2 e}, \forall T \in \Re$ we have

$$
\begin{aligned}
\left\|(h x)_{T}\right\|_{2} & \leq \gamma_{1}\left\|x_{T}\right\|_{2}+\alpha_{1} \\
\left\langle x_{T} \mid(h x)_{T}\right\rangle & \geq \delta_{1}\left\|x_{T}\right\|_{2}^{2}+\beta_{1} \\
\left\langle x_{T} \mid(f x)_{T}\right\rangle & \geq \delta_{2}\left\|(f x)_{T}\right\|_{2}^{2}+\beta_{2} .
\end{aligned}
$$

If $\delta_{1}+\delta_{2}>0$, then $u_{1}, u_{2} \in \mathcal{L}_{2} \Rightarrow e_{1}, e_{2}, y_{1}, y_{2} \in \mathcal{L}_{2}$. Furthermore, if $\alpha_{1}, \beta_{1}, \beta_{2}$ are zero, then the map from $\left(u_{1}, u_{2}\right)$ to $\left(e_{1}, e_{2}, y_{1}, y_{2}\right)$ is finite-gain $\mathcal{L}_{2}$ stable.

Proof. See $[12,33]$.

Note that if the operator $h$ is $\mathcal{L}_{2}$ stable, then the finite gain condition (13) is automatically satisfied. Also, if $h$ is strictly passive and $f$ is passive, then conditions (14) and (15) are satisfied with $\delta_{2}=0, \delta_{1}>0$.

For the AWBT stability analysis problem, as we will see in $\S 3, h$ is a fixed, stable, LTI system with transfer function $H(s)$ and $f$ belongs to a class of sector bounded nonlinearities with a specified diagonal structure. We will be interested in developing stability conditions for the entire class of $f$. Such a problem is referred to as the absolute stability problem. Application of the passivity theorem will lead to sufficient conditions for stability which can be potentially conservative. This is because Theorem 1 assumes $f$ to be any arbitrary operator satisfying (15), whereas, in reality, $f$ has some additional structural properties. In this case, we can apply multiplier theory $[4,5,33]$ to get less conservative conditions for stability by using this additional information about $f$. 


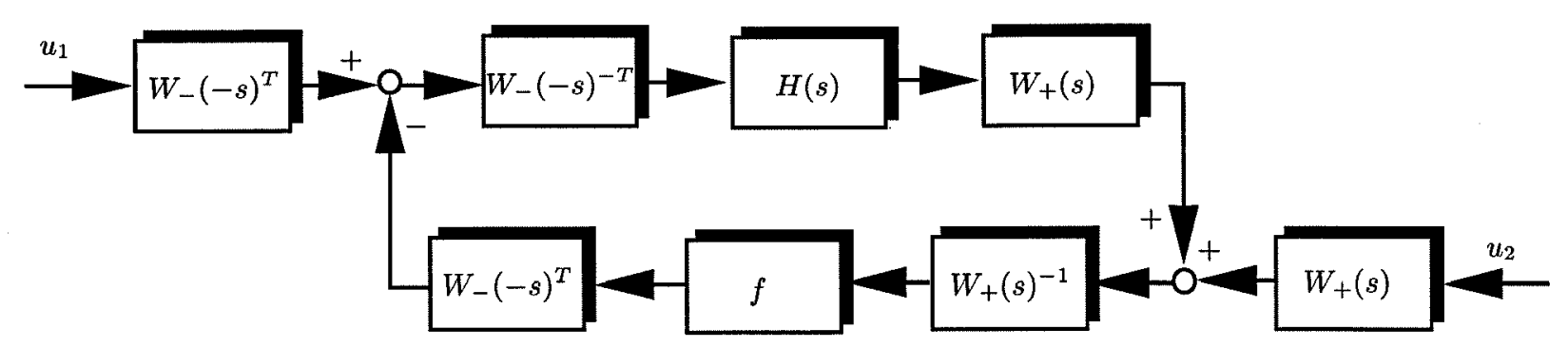

Figure 4: The passivity theorem with multipliers

The basic idea behind multiplier theory is that by multiplying the operators $h$ and $f$ by appropriately chosen multipliers, the product can be modified to satisfy the conditions of Theorem 1. Consider Figure 4 which is obtained from Figure 3 by pre- and post-multiplying $H(s)$ by $W_{-}(-s)^{-T}$ and $W_{+}(s)$ respectively, and correspondingly, pre- and post-multiplying $f$ by $W_{+}(s)^{-1}$ and $W_{-}(-s)^{T}$, and the inputs $u_{1}$ and $u_{2}$ by $W_{-}(-s)^{T}$ and $W_{+}(s)$. If we assume that $W_{+}(s), W_{-}(-s)$ are stable, proper and minimum phase with proper inverses, then the stability of the systems in Figures 3 and 4 are equivalent. Applying Theorem 1 then gives the following result.

Corollary 1 Consider the feedback system shown in Figure 4. Assume that for any $u_{1}, u_{2} \in$ $\mathcal{L}_{2}$, all the signals in the system are well-defined and belong to $\mathcal{L}_{2 e}$. Then, the system is $\mathcal{L}_{2}$ stable if

1. $\exists W_{+}(s), W_{-}(-s)$ which are stable and proper with stable and proper inverses;

2. $W_{+}(s) H(s) W_{-}(-s)^{-T}$ is $\mathcal{L}_{2}$ stable; and

3. $\exists$ constants $\beta_{1}, \beta_{2}, \delta_{1}, \delta_{2}$ such that $\forall x \in \mathcal{L}_{2 e}, \forall T \in \Re$, we have

$$
\begin{aligned}
\left\langle x_{T} \mid(\tilde{h} x)_{T}\right\rangle & \geq \delta_{1}\left\|x_{T}\right\|_{2}^{2}+\beta_{1} \\
\left\langle x_{T} \mid(\tilde{f} x)_{T}\right\rangle & \geq \delta_{2}\left\|(\tilde{f} x)_{T}\right\|_{2}^{2}+\beta_{2} \\
\delta_{1}+\delta_{2} & >0
\end{aligned}
$$

where $\tilde{h}, \tilde{f}$ are the operators $W_{+}(s) H(s) W_{-}(-s)^{-T}$ and $W_{-}(-s)^{T} \circ f \circ W_{+}(s)^{-1}$ respectively (symbol "o" denotes composition).

Remark 2 In most problems of interest, as also in the AWBT analysis problem of $\S 3$, Corollary 1 is applied with $\delta_{2}=0$. This will be assumed to be the case in the rest of the paper. In that case, using (10), we can conclude that conditions (16) and (18) above are equivalent to the existence of $\delta_{1}>0$ such that

$$
\begin{gathered}
W_{+}(j \omega) H(j \omega) W_{-}(j \omega)^{-*}+W_{-}(j \omega)^{-1} H(j \omega)^{*} W_{+}(j \omega)^{*} \geq 2 \delta_{1} I, \quad \forall \omega \in \Re \\
\Longleftrightarrow W(j \omega) H(j \omega)+H(j \omega)^{*} W(j \omega)^{*} \geq \delta^{\prime} I, \quad \forall \omega \in \Re, \text { for some } \delta^{\prime}>0 .
\end{gathered}
$$


In (19), $W(s)=W_{-}(s) W_{+}(s)$ is commonly referred to as the stability multiplier. Since $W_{-}(-s)$ is stable, $W(s)=W_{-}(s) W_{+}(s)$ is in general unstable or equivalently, non-causal. Note that if we are given the state-space representation of $W(s) H(s)$, then we can use Lemma 1 to check condition (19) in terms of the state-space matrices of $W(s) H(s)$.

Thus, we see that stability analysis using multipliers involves finding a multiplier $W(s)$ such that it can be factorized into $W_{-}(s) W_{+}(s)$, with $W_{-}(s), W_{+}(s)$ satisfying conditions 1 and 2 of Corollary 1 and (17), and $W(s), H(s)$ satisfying (19).

The significance of the multiplier approach to stability analysis discussed in this section is that a host of well-known, seemingly different stability analysis tests can be shown to be special cases of Corollary 1 for particular choices of the multiplier $W(s)$ (see $[4,5,28]$ ). These special cases include the Circle Criterion, the Off-axis Circle Criterion and the Popov Criterion in the SISO case [28], and upper bounds on $\mu$ [26] for multivariable systems with structured, (mixed) real/complex and parametric uncertainties [4,5]. Moreover, given the multiplier $W(s)$ establishing stability of the closed-loop, the corresponding quadratic Lyapunov function establishing stability for the closed-loop can be explicitly constructed [3].

With these preliminaries, we now consider the AWBT stability analysis problem.

\section{Stability Analysis of AWBT Control Systems}

Consider the AWBT compensated system of Figure 2, where $\hat{P}(s)$ and $\hat{K}(s)$, partitioned according to their inputs and outputs, are given respectively by (2) and (5). We will assume that $P_{22}(\infty)=0$ to ensure well-posedness of the linear interconnection in Figure 1(a). It is easy to verify that Figure 2 can be rearranged in the form shown in Figure 5 with

$$
M(s)=\left[\begin{array}{cc}
(V(s)-I) P_{32}(s)-U(s) P_{22}(s) & U(s) P_{21}(s) \\
-P_{12}(s) & P_{11}(s)
\end{array}\right]=\left[\begin{array}{cc}
M_{11}(s) & M_{12}(s) \\
M_{21}(s) & M_{22}(s)
\end{array}\right] .
$$

$N$ is the generic input nonlinearity which represents either component-wise actuator sat-

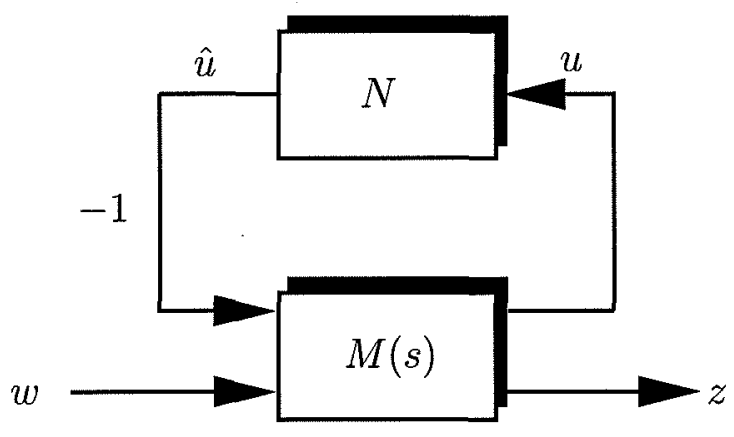

Figure 5: Interconnection for AWBT stability analysis

uration, relay, dead-zone, hysteresis, etc. (input limitation) or an override, mode selection scheme/switching logic (input substitution). We will assume that the $M_{11}(s)-N$ loop is well-posed. This can be ensured, for instance, by assuming that $M_{11}(\infty)+M_{11}(\infty)^{T}>0$. 
Exact stability analysis, i.e., development of non-conservative conditions which are both sufficient and necessary for stability of the system in Figure 5, for a given nonlinearity $N$ (for example, saturation) is, in general, a difficult problem. On the other hand, as we will see in $\S 3.1$, firstly, the nonlinearity $N$ can be assumed to be memoryless, i.e., its output at any time depends only on its input at the present time. Secondly, bounds on its input-output map (for example, sector bounds) can be easily derived. Based on these two facts, we can cover $N$ by a class of sector bounded memoryless nonlinearities having the same structure as $N$. We can then apply results from absolute stability theory $[12,23]$ to develop sufficient conditions which guarantee stability for the entire class of $N$ rather than $N$ itself. This has probably been the most common approach for analyzing AWBT stability (see for example $[8,9,19,20,21,22])$, the reason being that it greatly simplifies the nonlinear analysis.

The unavoidable price paid for this simplification is conservatism since the resulting conditions ensure stability not only for $N$ but also for all nonlinear maps with the given structure and sector bounds. In $\S 3.2$, we will see how we can reduce this conservatism by applying concepts from multiplier theory to incorporate additional properties of $N$.

\subsection{Sector bounds on the nonlinearity $N$}

In this section, we derive sector bounds for common input limitation and substitution nonlinearities. We begin by defining a sector condition on a nonlinearity.

Definition 3 Let $f: \Re^{n} \times \Re \longrightarrow \Re^{n}$ with $f(0, t)=0 \forall t \geq 0$ be a memoryless (possibly time-varying) diagonal nonlinearity $f=\operatorname{diag}\left\{f_{1}, \ldots, f_{n}\right\}$. We say that $f \in \operatorname{sector}\left[K_{1}, K_{2}\right]$, with $K_{1}=\operatorname{diag}\left(K_{11}, \ldots, K_{1 n}\right), K_{2}=\operatorname{diag}\left(K_{21}, \ldots, K_{2 n}\right), K_{2}-K_{1}>0$ if

$$
K_{1 i} x_{i}^{2} \leq x_{i} f_{i}\left(x_{i}, t\right) \leq K_{2 i} x_{i}^{2}, \text { for all } x_{i} \in \Re, t \geq 0, i=1,2, \ldots, n \text {. }
$$

Consider the interconnection in Figure 3, where $h$ is assumed to be a fixed LTI system with transfer function $H(s)$ and $f$ is a diagonal nonlinearity lying in the sector $\left[K_{1}, K_{2}\right]$, with $K_{1}, K_{2}$ as in definition 3. Suppose we apply a negative feedforward of $K_{1}$ and a positive feedback of $\left(K_{2}-K_{1}\right)^{-1}$ to the nonlinearity $f$, and correspondingly, we apply a negative feedback of $K_{1}$ and a positive feedforward of $\left(K_{2}-K_{1}\right)^{-1}$ to $H(s)$ as shown in Figure 6. This is a well-known loop transformation (see [12, §VI.9]) from Figure 3 to the equivalent interconnection in Figure 6 . The resulting nonlinear subsystem $\tilde{f}$ is a diagonal operator $\tilde{f}=\operatorname{diag}\left\{\tilde{f}_{1}, \ldots, \tilde{f}_{n}\right\}$ where $\tilde{f}_{i}$ lies in the sector $[0, \infty]$ (see $[12, \S$ VI.9] for details) and satisfies the sector condition

$$
x_{i} \tilde{f}_{i}\left(x_{i}, t\right) \geq 0, \forall x_{i} \in \Re, t \geq 0, i=1,2, \ldots, n
$$

i.e., the graph of $\tilde{f}_{i}\left(x_{i}, t\right)$ vs. $x_{i}$ lies in the first and third quadrants. We may note that, by definition, $\tilde{f}$ is passive and Remark 2 from $\S 2.2$ applies in this case. The linear subsystem is given by

$$
\tilde{H}(s)=\left(K_{2}-K_{1}\right)^{-1}\left(I+K_{2} H(s)\right)\left(I+K_{1} H(s)\right)^{-1} .
$$

The reason for introducing this loop transformation is that, as shown in [32], applying the passivity theorem with multipliers to the transformed system in Figure 6 gives potentially less conservative stability conditions than those resulting from its application to the original system in Figure 3. We will use this loop transformation in the AWBT stability analysis problem. 


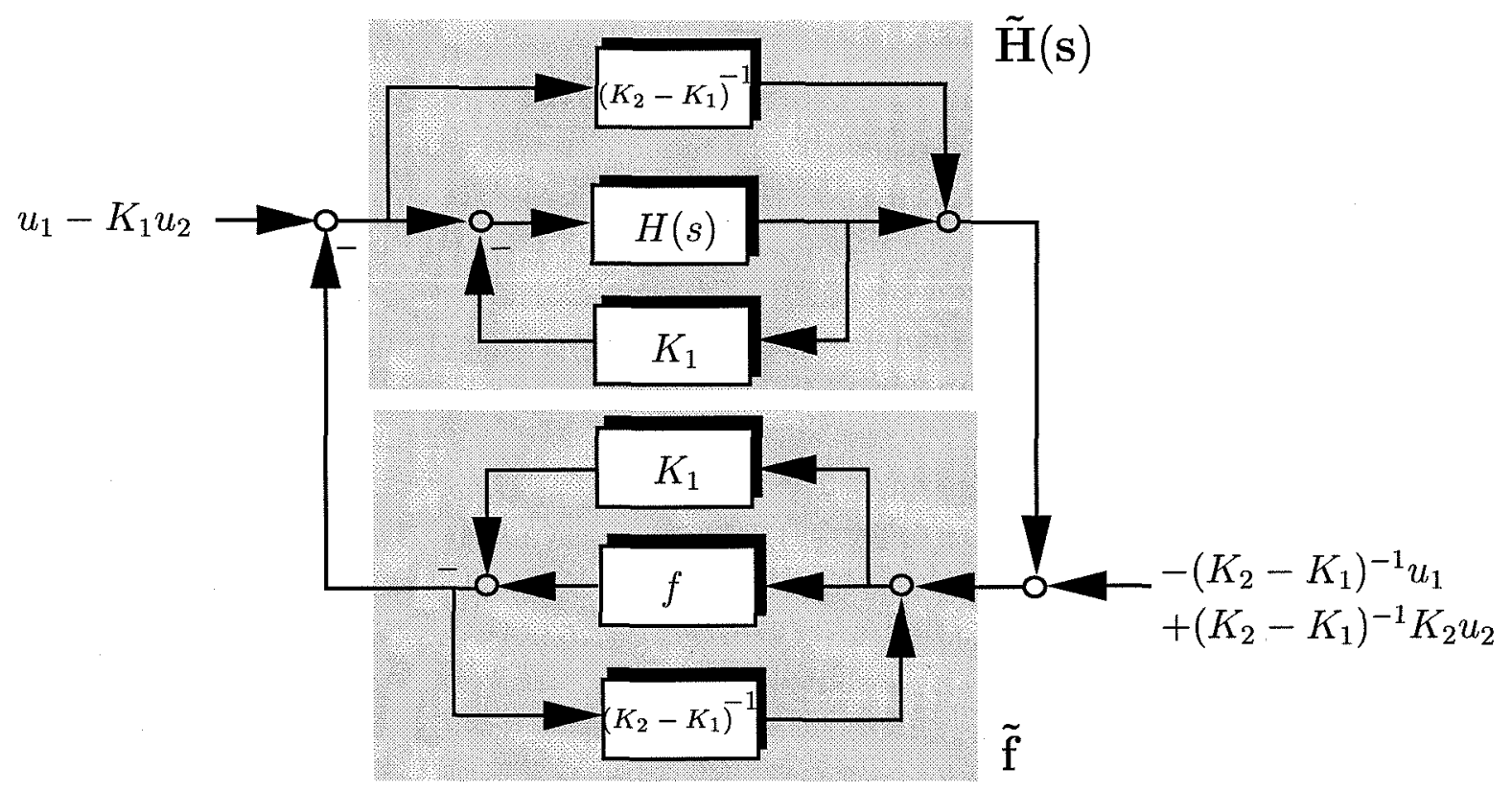

Figure 6: A loop transformation

\subsubsection{Limitations}

The most common example of an input limitation is actuator saturation (see Figure 7). Multivariable actuator saturation can be described by a memoryless, time-invariant, diagonal operator $N=\operatorname{diag}\left\{N_{1}, \ldots, N_{n_{u}}\right\}$, where the $N_{i}$ 's are defined as follows:

$$
\begin{aligned}
N_{i}\left(u_{i}\right) & =\operatorname{sat}\left(u_{i}\right) \\
& = \begin{cases}u_{i, \min } & \text { if } u_{i}<u_{i, \min } \\
u_{i} & \text { if } u_{i, \min } \leq u_{i} \leq u_{i, \max } \\
u_{i, \max } & \text { if } u_{i}>u_{i, \max } .\end{cases}
\end{aligned}
$$

It is easy to verify that

$$
0 \leq u_{i} N_{i}\left(u_{i}\right) \leq u_{i}^{2}, \quad \text { for all } u_{i} \in \Re
$$

and hence $N \in \operatorname{sector}[0, I]$ as shown in Figure 7(a). We note that both the identity operator $N=I$ and the zero operator $N=0$ are included in the sector. However, if the controller output $u_{i}$ can be bounded in magnitude, then the zero operator need not be included and we can take $K_{1} \neq 0$ in (21). This will give a tighter sector bound for $N_{i}$ as shown in Figure 7(b).

Other input nonlinearities such as dead-zones, relays, relays with dead-zones and hysteresis can also be covered by sectors in a similar manner. Note that except for hysteresis, all these nonlinearities are time-invariant, whereas the sector bounds include nonlinearities which are allowed to be arbitrarily time-varying.

\subsubsection{Substitutions}

Substitution mechanisms arise from the use of overrides or logic schemes which select the plant input $\hat{u}$ from among the outputs of a "bank" of controllers, each designed to achieve 


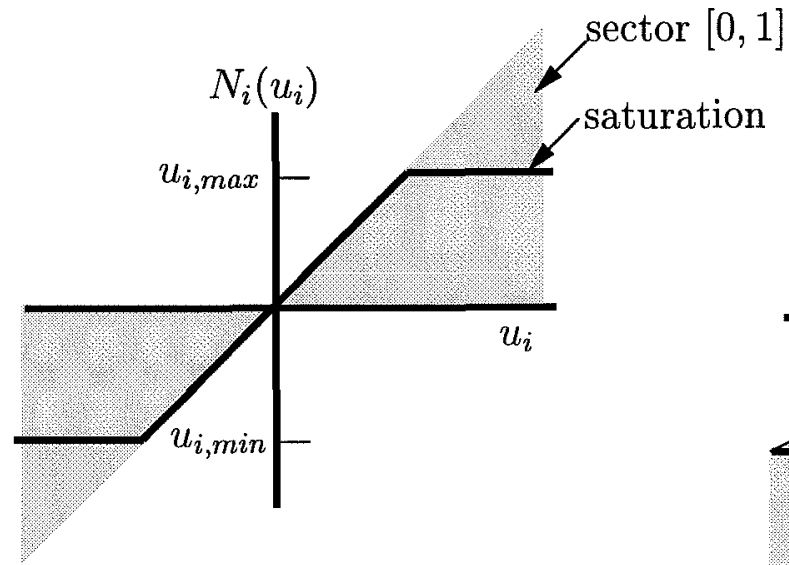

(a)

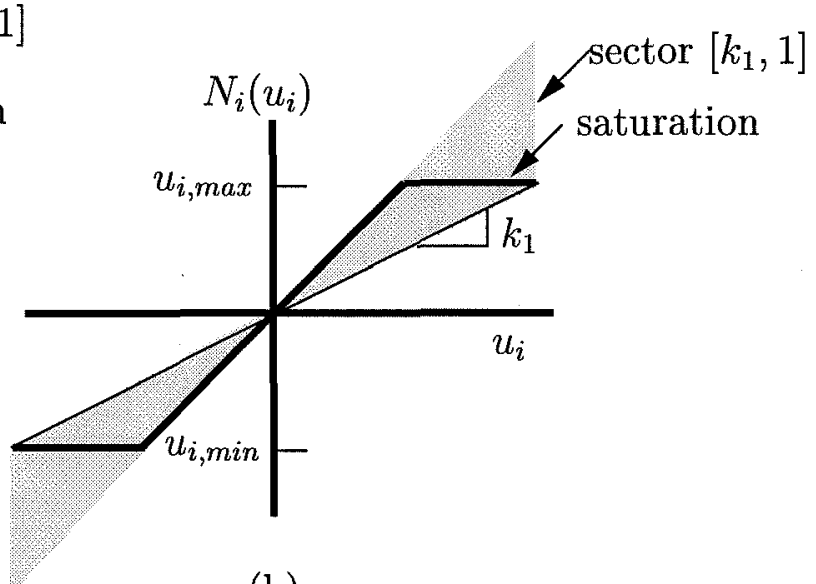

(b)

Figure 7: Sector bounds on the saturation nonlinearity $N$

a different closed-loop characteristic. Commonly employed logic blocks are "min" selectors and "max" selectors which respectively select the minimum and maximum input as their output. Combinations of min-max selectors shown in Figure 8(a) are often used to enforce

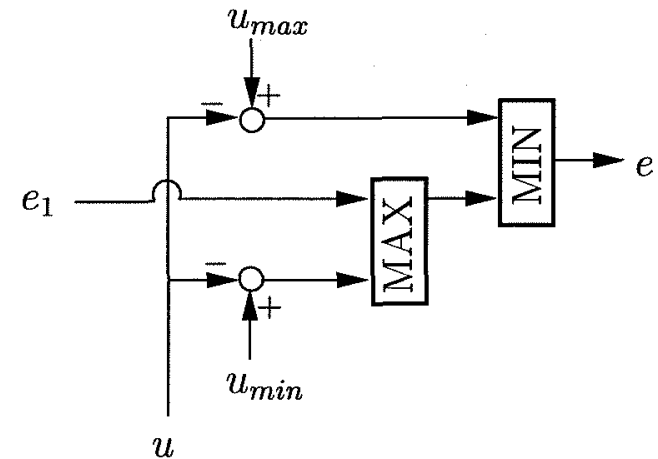

(a)

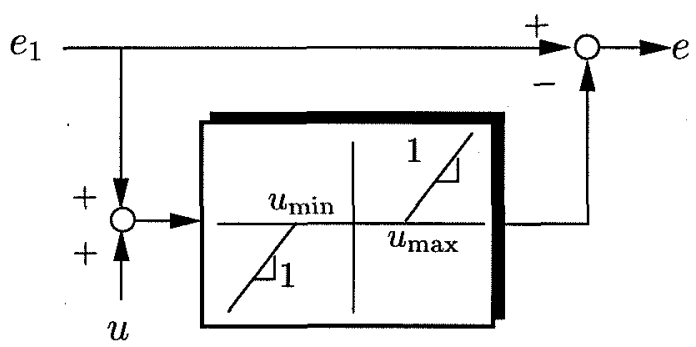

(b)

Figure 8: a) A combination of "min-max" selectors; b) its equivalent representation using a dead-zone nonlinearity.

upper and lower bounds on some variable, for example, $u$ in Figure 8(a). It is easy to verify (see $[19,20]$ ) that this min-max selector can be equivalently represented by using a deadzone nonlinearity as shown in Figure 8(b). As discussed in $§ 3.1 .1$, this dead-zone can then be covered by the sector $[0,1]$.

Min and max selectors are only special cases of a "generic" selector which selects one of its inputs as its output. If we assume that the mechanism which determines which input is selected is completely unspecified or arbitrary, then the generic selector can be approximated by an arbitrarily time-varying memoryless switching nonlinearity $N$. For example, if the selector has two inputs $u_{1}, u_{2}$ and chooses one of them as its output $\hat{u}$, then this selector can 
be approximated as follows:

$$
\begin{aligned}
\hat{u} & =N\left(u_{1}, u_{2}, t\right) \\
& =u_{1}+n(t)\left(u_{2}-u_{1}\right)
\end{aligned}
$$

where $n(t) \in$ sector $[0,1]$ is an arbitrarily time-varying memoryless parameter. $n(t)=0$ and 1 give respectively the outputs $u_{1}$ and $u_{2}$. Selectors with more than two inputs can be modeled by decomposing them into a series of two-input selectors. The resulting multiple nonlinearities can then be arranged in a diagonal form. Sector bounds for combinations of selectors and other nonlinearities can be worked out using the same basic principles.

\subsection{Sufficient Conditions for AWBT Stability}

As discussed at the beginning of $\S 3$, we will derive sufficient conditions which ensure stability of the system in Figure 5 for all $N$ with a given structure and sector bounds. A problem of this type was originally formulated by Lur'e [25] and is known as the absolute stability problem. The basic idea is to derive conditions on the linear subsystem $M$ such that the closed loop system in Figure 5 is stable for all nonlinearities $N$ belonging to a certain class. Theorem 1 and Corollary 1 in $\S 2.2$ form the basis of the stability results that follow. We begin with the most general case by allowing the nonlinearity $N$ to be arbitrarily time-varying. We then successively impose more restrictions on the nonlinearity $N$. Correspondingly, we modify the choice of the multiplier $W(s)$ in Corollary 1 to get less conservative stability conditions. In all cases, we show how the multiplier $W(s)$ establishing stability can be explicitly constructed from the feasible solution of a set of convex conditions involving LMIs.

\subsubsection{Memoryless Time-Varying Nonlinearities}

We begin by defining the set $\mathcal{N}_{T V}$ of all allowable structured nonlinearities $N$.

$$
\begin{aligned}
\mathcal{N}_{T V}= & \left\{N: \Re^{n_{u}} \times \Re \rightarrow \Re^{n_{u}} \mid N(0, t)=0 \forall t \geq 0,\right. \\
& \left.N=\operatorname{diag}\left\{N_{1}, N_{2}, \ldots, N_{n_{u}}\right\}, N_{i} \in \operatorname{sector}[0,1]\right\} .
\end{aligned}
$$

The nonlinearities in $\mathcal{N}_{T V}$ are memoryless and are allowed to be arbitrarily time-varying. Here we consider only the sector $[0, I]$. Conditions for other sector bounds can be derived similarly. $\mathcal{N}_{T V}$ typically includes input nonlinearities such as those represented by (25) which model generic selectors with no pre-specified switching logic. Applying Corollary 1 to Figure 5 gives us the following result.

Theorem 2 (Multiloop Circle Criterion) The AWBT system in Figure 5 is $\mathcal{L}_{2}$ stable for all $N \in \mathcal{N}_{T V}$ if

1. $A-H_{1} C$ has all eigenvalues in the open left-half complex plane;

2. $\hat{P}$ in equation (2) is asymptotically stable; and

3. $\exists W=\operatorname{diag}\left(W_{1}, W_{2}, \ldots, W_{n_{u}}\right) \in \Re^{n_{u} \times n_{u}}$ with $W>0$ and $\delta_{1}>0$ such that

$$
W M_{11}(j \omega)+M_{11}^{*}(j \omega) W+2 W \geq \delta_{1} I, \quad \forall \omega \in \Re .
$$


Furthermore, if $M_{11}(s)=\left[\begin{array}{c|c}\tilde{A} & \tilde{B} \\ \hline \tilde{C} & \tilde{D}\end{array}\right]$, then (27) above can be equivalently checked via the existence of a symmetric matrix $Q=Q^{T}>0, \delta_{1}>0$ such that the following LMI in $Q, W, \delta_{1}$ is satisfied

$$
\left[\begin{array}{cc}
\tilde{A}^{T} Q+Q \tilde{A} & Q \tilde{B}-\tilde{C}^{T} W \\
\tilde{B}^{T} Q-W \tilde{C} & \delta_{1} I-2 W-W \tilde{D}-\tilde{D}^{T} W
\end{array}\right] \leq 0
$$

Proof. By assumption, the loop $M_{11}(s)-N$ is well-posed. For $\mathcal{L}_{2}$ stability, it is enough to show that $M(s)$ is $\mathcal{L}_{2}$ stable and that the $M_{11}(s)-N$ loop is asymptotically stable. Condition 1 of the Theorem and (6) imply that $\hat{K}(s)$ is asymptotically stable. Together with condition 2 above and (20), this implies that $M(s)$ (and hence $M_{11}(s)$ ) is asymptotically stable.

Next, consider the loop $M_{11}(s)-N$, where, $N \in$ sector $[0, I]$. Applying the loop transformation of Figure 6, we transform the diagonal nonlinearity $N$ to a diagonal nonlinearity $\tilde{N}$ with $\tilde{N}_{i} \in$ sector$[0, \infty]$, and correspondingly, we transform $M_{11}(s)$ to $\tilde{M}_{11}(s)=M_{11}(s)+I$. Since $\tilde{N}_{i} \in$ sector $[0, \infty], \tilde{N}$ is passive. Corollary 1 can now be applied to the $\tilde{M}_{11}(s)-\tilde{N}$ loop with $H(s)=\tilde{M}_{11}(s), f=\tilde{N}$.

Since $\tilde{N}$ is an arbitrarily time-varying, memoryless nonlinearity, an appropriate multiplier for this case is $W(s)=W>0$ where $W=\operatorname{diag}\left(W_{1}, W_{2}, \ldots, W_{n_{u}}\right) \in \Re^{n_{u} \times n_{u}}$, with $W_{+}(s)=$ $W, W_{-}(s)=I$ (see $\left.[4,5]\right)$. This multiplier clearly satisfies (17) with $\delta_{2}=0$ (see Remark 2) and conditions 1 and 2 of Corollary 1. (16) and (18) can be checked via (19) as follows:

$$
\begin{gathered}
W \tilde{M}_{11}(j \omega)+\tilde{M}_{11}^{*}(j \omega) W \geq \delta_{1} I, \quad \forall \omega \in \Re \\
\Leftrightarrow W M_{11}(j \omega)+M_{11}^{*}(j \omega) W+2 W \geq \delta_{1} I, \quad \forall \omega \in \Re
\end{gathered}
$$

which establishes (27). Further, if $M_{11}(s)=\left[\begin{array}{c|c}\tilde{A} & \tilde{B} \\ \hline \tilde{C} & \tilde{D}\end{array}\right]$, then $W \tilde{M}_{11}(s)=\left[\begin{array}{c|c}\tilde{A} & \tilde{B} \\ \hline W \tilde{C} & W+W \tilde{D}\end{array}\right]$.

Lemma 1 then establishes (28).

We may note that the stability multiplier $W(s)=W$ is directly computed once the LMI (28) is solved.

Theorem 2 generalizes the AWBT stability results from $[2,8,9,15,22]$ which were derived using small-gain arguments, generalized $\mu$ upper bounds, a version of the multiloop Circle Criterion and describing functions. The stability result in [22] corresponds to choosing the multiplier $W$ to be the identity matrix. However, the result from [22] can be potentially conservative since it does not account for the diagonal structure of $N$. The results in [8, $9,15]$ correspond to choosing the multiplier $W$ to be a constant, diagonal, positive-definite complex matrix. Although choosing $W$ to be complex facilitates computation of the stability conditions in $[8,9,15]$, it does not account for the fact that the nonlinearity $N$ is real.

\subsubsection{Memoryless Time-Invariant (Static) Nonlinearities}

In the previous section, the set $\mathcal{N}_{T V}$ included arbitrarily time-varying memoryless nonlinearities. A large class of input nonlinearities such as saturation, relay, dead-zone, relay with dead-zone, etc. are memoryless and time-invariant, i.e., static. Almost all previously reported AWBT stability analysis results $[9,8,15,19,20,21,22]$ model these static nonlinearities as time-varying to simplify the analysis problem. The resulting stability conditions, 
as also those obtained from Theorem 2, are potentially extremely conservative in such cases. This conservatism can be reduced by appropriately modifying the choices of $W_{+}(s)$ and $W_{-}(s)$ such that the time-invariance property of $N$ is taken into account.

Let us define the class of memoryless time-invariant nonlinearities as follows:

$$
\mathcal{N}_{T I}=\left\{N: \Re^{n_{u}} \rightarrow \Re^{n_{u}} \mid N(0)=0, N=\operatorname{diag}\left\{N_{1}, N_{2}, \ldots, N_{n_{u}}\right\}, N_{i} \in \operatorname{sector}[0,1]\right\} .
$$

Using a variety of techniques, Popov (1961) [27], Zames (1966) [31] and Brockett and Willems (1965) [7] have shown that the appropriate multiplier for this case, with a scalar nonlinearity $\left(n_{u}=1\right)$, is $W(s)=1+W s, W \geq 0$, with $W_{+}(s)=1+W s, W_{-}(s)=1$. As we will see, with a multivariable diagonal nonlinearity $N$ belonging to the set $\mathcal{N}_{T I}$, the appropriate multiplier is the following:

$$
W(s)=X+s W \quad \text { with } W_{+}(s)=(X+s W), \quad W_{-}(s)=I
$$

where $W=\operatorname{diag}\left(W_{1}, W_{2}, \ldots, W_{n_{u}}\right) \in \Re^{n_{u} \times n_{u}}, X=\operatorname{diag}\left(X_{1}, X_{2}, \ldots, X_{n_{u}}\right) \in \Re^{n_{u} \times n_{u}}, W \geq 0$, $X>0$. Note that although $(X+s W)$ is not proper, it can be obtained as the limit

$$
X+s W=\lim _{n \rightarrow \infty} \frac{1}{1+\frac{s}{n}}(X+s W)
$$

where $\frac{1}{1+\frac{s}{n}}(X+s W)$ is the appropriate proper multiplier satisfying the conditions of Corollary 1 . We will need the following lemma to prove AWBT stability with static input nonlinearities.

Lemma 2 Let $f=\operatorname{diag}\left\{f_{1}, f_{2}, \ldots, f_{n}\right\}$ be a memoryless, time-invariant, diagonal nonlinearity $f: \Re^{n} \longrightarrow \Re^{n}$, with $f_{i} \in$ sector $[0, \infty]$. Let $W(s)=X+s W, X=\operatorname{diag}\left(X_{1}, X_{2}, \ldots, X_{n}\right) \in$ $\Re^{n \times n}, W=\operatorname{diag}\left(W_{1}, W_{2}, \ldots, W_{n}\right) \in \Re^{n \times n}$ with $W \geq 0, X>0$. Then $f \circ W(s)^{-1}$ is passive.

Proof. The proof is a multivariable extension of the proof of Lemma 2 in [31]. Since $f_{i} \in \operatorname{sector}[0, \infty], f$ is passive, i.e., it satisfies

$$
\left\langle x_{T} \mid(f x)_{T}\right\rangle \geq 0, \forall x \in \Re^{n}, T \geq 0 .
$$

We need to show that $f \circ W(s)^{-1}$ is passive, i.e., that it satisfies

$$
\left\langle x_{T} \mid\left(f \circ W(s)^{-1} x\right)_{T}\right\rangle \geq 0, \forall x \in \Re^{n}, T \geq 0 .
$$

Now,

$$
\begin{aligned}
\left\langle x_{T} \mid\left(f \circ W(s)^{-1} x\right)_{T}\right\rangle & \left.=\left\langle(W(s) y)_{T} \mid(f y)_{T}\right\rangle \quad \text { (substituting } y=W(s)^{-1} x\right) \\
& =\int_{0}^{T}(X y(t)+W \dot{y}(t))^{T} f(y(t)) d t \\
& =\int_{0}^{T} y(t)^{T} X f(y(t)) d t+\int_{0}^{T} \dot{y}(t)^{T} W f(y(t)) d t \\
& \geq \int_{0}^{y(t)} f(y(t))^{T} W d y \geq 0 .
\end{aligned}
$$


The last two inequalities follow from the fact that $f$ is diagonal, i.e. $f=\operatorname{diag}\left\{f_{1}, \ldots, f_{n}\right\}$ with $f_{i} \in \operatorname{sector}[0, \infty]$ and $W \geq 0, X>0$ are diagonal matrices.

The following theorem, a multivariable extension of the classical scalar Popov criterion, states conditions for AWBT stability with static input nonlinearities.

Theorem 3 (Multivariable Popov Criterion) The AWBT system in Figure 2 is $\mathcal{L}_{2}$ stable for all $N \in \mathcal{N}_{T I}$ if

1. $A-H_{1} C$ has all eigenvalues in the open left-half complex plane;

2. $\hat{P}$ in equation (2) is asymptotically stable; and

3. $\exists X=\operatorname{diag}\left(X_{1}, X_{2}, \ldots, X_{n_{u}}\right) \in \Re^{n_{u} \times n_{u}}, W=\operatorname{diag}\left(W_{1}, W_{2}, \ldots, W_{n_{u}}\right) \in \Re^{n_{u} \times n_{u}}$ with $W \geq 0, X>0$ and $\delta_{1}>0$ such that if $M_{11}(s)=\left[\begin{array}{c|c}\tilde{A} & \tilde{B} \\ \hline \tilde{C} & \tilde{D}\end{array}\right]$, then

$$
\begin{gathered}
(X+j \omega W)\left(M_{11}(j \omega)-\tilde{D}\right)+\left(M_{11}^{*}(j \omega)-\tilde{D}^{T}\right)(X-j \omega W) \\
+X \tilde{D}+\tilde{D}^{T} X+2 X \geq \delta_{1} I, \quad \forall \omega \in \Re .
\end{gathered}
$$

Furthermore, (31) above can be equivalently checked via the existence of a symmetric matrix $Q=Q^{T}>0, \delta_{1}>0$ such that the following LMI in $Q, W, X, \delta_{1}$ is satisfied

$$
\left[\begin{array}{cc}
\tilde{A}^{T} Q+Q \tilde{A} & Q \tilde{B}-\tilde{C}^{T} X-\tilde{A}^{T} \tilde{C}^{T} W \\
\tilde{B}^{T} Q-X \tilde{C}-W \tilde{C} \tilde{A} & \delta_{1} I-W \tilde{C} \tilde{B}-\tilde{B}^{T} \tilde{C}^{T} W-X \tilde{D}-\tilde{D}^{T} X-2 X
\end{array}\right] \leq 0 .
$$

Proof. As in the proof of Theorem 2, we see that $M(s)$ is stable and the $M_{11}(s)-N$ loop is well-posed. Also, as in the proof of Theorem 2, the $M_{11}(s)-N$ loop can be transformed to the $\tilde{M}_{11}(s)-\tilde{N}$ loop with $\tilde{M}_{11}(s)=M_{11}(s)+I, \tilde{N}=\operatorname{diag}\left\{\tilde{N}_{1}, \ldots, \tilde{N}_{n_{u}}\right\}, \tilde{N}_{i} \in$ sector $[0, \infty]$ with $\tilde{N}$ passive. Corollary 1 can now be applied to the $\tilde{M}_{11}(s)-\tilde{N}$ loop with $H(s)=\tilde{M}_{11}(s)$, $f=\tilde{N}, W(s)=X+s W, W_{+}(s)=X+s W, W_{-}(s)=I$, where $W=\operatorname{diag}\left(W_{1}, W_{2}, \ldots, W_{n_{u}}\right) \in$ $\Re^{n_{u} \times n_{u}}, X=\operatorname{diag}\left(X_{1}, X_{2}, \ldots, X_{n_{u}}\right) \in \Re^{n_{u} \times n_{u}}, W \geq 0, X>0$.

By Lemma 2, $N \circ W(s)^{-1}$ is passive and hence (17) is satisfied with $\delta_{2}=0$ (see Remark 2). (16) and (18) can be checked via (19) as follows:

$$
\begin{gathered}
(X+j \omega W) \tilde{M}_{11}(j \omega)+\tilde{M}_{11}^{*}(j \omega)(X-j \omega W) \geq \delta_{1} I, \quad \forall \omega \in \Re \\
\Leftrightarrow(X+j \omega W)\left(M_{11}(j \omega)-\tilde{D}\right)+\left(M_{11}^{*}(j \omega)-\tilde{D}^{T}\right)(X-j \omega W) \\
+X \tilde{D}+\tilde{D}^{T} X+2 X \geq \delta_{1} I, \quad \forall \omega \in \Re
\end{gathered}
$$

which establishes (31). Note that the above inequality can be rigorously derived using the multiplier $W(s)=\frac{1}{1+\frac{s}{n}}(X+s W)$ and taking the limit as $n \rightarrow \infty$. Furthermore, it can be verified that $(X+s W)\left(M_{11}(s)-\tilde{D}\right)=\left[\begin{array}{c|c}\tilde{A} & \tilde{B} \\ \hline X \tilde{C}+W \tilde{C} \tilde{A} & W \tilde{C} \tilde{B}\end{array}\right]$. (32) then follows from Lemma 1 and this completes the proof.

As in Theorem 2, we may note that the multiplier $W(s)=X+s W$ establishing stability is explicitly determined once we compute a feasible solution to the LMI (32). 
In $[19,20,21]$, the Popov criterion was used to ascertain stability of SISO anti-reset windup PI control systems. However, in their work, the scalar Popov parameter $W \geq 0$ could only be deduced graphically. Hence, it was not clear how their analysis could be extended to the MIMO case. Theorem 3 generalizes their analysis technique to the multivariable case by giving a sufficient condition for AWBT stability in terms of the feasibility of an LMI in the Popov matrix parameters $W \geq 0, X>0$, the symmetric matrix $Q>0$ and the scalar $\delta_{1}>0$. Hence, the existence (or absence thereof) of a Popov multiplier establishing AWBT stability can be readily determined via a convex LMI condition.

\subsubsection{Monotonic Slope-Restricted Static Nonlinearities}

Several input nonlinearities, in addition to being memoryless and time-invariant, are also (odd) monotonic and/or slope-restricted. Examples include saturation, dead-zone, relay and relay with dead-zone. To the best of our knowledge, there has been no attempt to incorporate these additional properties of the input nonlinearities to get improved AWBT stability conditions. As we will see, it is possible to exploit these properties, by appropriately choosing the multiplier $W(s)$, to get less conservative stability conditions.

Definition 4 Let $f: \Re^{n} \rightarrow \Re^{n}$ with $f(0)=0$ be a static diagonal nonlinearity $f=$ $\operatorname{diag}\left\{f_{1}, f_{2}, \ldots, f_{n}\right\} . f$ is said to be monotone non-decreasing if

$$
\left(x_{1 i}-x_{2 i}\right)\left(f_{i}\left(x_{1 i}\right)-f_{i}\left(x_{2 i}\right)\right) \geq 0, \quad \forall x_{1 i}, x_{2 i} \in \Re, i=1,2, \ldots, n,
$$

and $f$ is said to be odd monotone non-decreasing if, in addition

$$
f(-x)=-f(x), \quad \forall x \in \Re^{n} .
$$

Definition 5 Let $f: \Re^{n} \rightarrow \Re^{n}$ with $f(0)=0$ be a static diagonal nonlinearity $f=$ $\operatorname{diag}\left\{f_{1}, f_{2}, \ldots, f_{n}\right\} . f$ is said to be incrementally inside (or slope-restricted in) sector $\left[K_{1}, K_{2}\right]$, with $K_{1}=\operatorname{diag}\left(K_{11}, \ldots, K_{1 n}\right), K_{2}=\operatorname{diag}\left(K_{21}, \ldots, K_{2 n}\right), K_{2}-K_{1}>0$ if

$$
K_{1 i} \leq \frac{f_{i}\left(x_{1 i}\right)-f_{i}\left(x_{2 i}\right)}{x_{1 i}-x_{2 i}} \leq K_{2 i}, \quad \forall x_{1 i}, x_{2 i} \in \Re, x_{1 i} \neq x_{2 i}, i=1,2, \ldots, n .
$$

It is easy to verify that the saturation nonlinearity $N_{i}$ of Figure 7 (a) satisfies (33) and (35) with $K_{1 i}=0, K_{2 i}=1$. Furthermore, if $u_{i, \min }=-u_{i, \max }$, then $N_{i}$ also satisfies (34).

Absolute stability of the feedback interconnection in Figure 3, where $h$ is a causal LTI system with transfer function $H(s)$ and $f$ is an (odd) monotonic, slope-restricted, static scalar nonlinearity, was originally studied by Zames and Falb (1968) [33]. The basic idea of the stability proof was to characterize the appropriate multiplier to be used in Corollary 1. The following theorem, a multivariable extension of the result from Zames and Falb (1968) [33], states conditions for AWBT stability with static slope-restricted (odd) monotone nonlinearities.

Theorem 4 Let $w_{i}(t), i=, 1,2, \ldots, n_{u}$ be the impulse response of a scalar LTI (possibly non-causal) operator on $t \in(-\infty, \infty)$ with

$$
\int_{-\infty}^{\infty}\left|w_{i}(t)\right| d t<X_{i}, X_{i}>0, \quad i=1,2, \ldots, n_{u} .
$$


Then the $A W B T$ system in Figure 2 is $\mathcal{L}_{2}$ stable for all $N \in \mathcal{N}_{\text {TI }}$ with $N$ being odd monotone non-decreasing and incrementally inside sector $[0, I]$ if

1. $A-H_{1} C$ has all eigenvalues in the open left-half complex plane;

2. $\hat{P}$ in equation (2) is asymptotically stable; and

3. $\exists w_{i}(t)$, with Fourier transform $W_{i}(j \omega)$, and $X_{i}>0, \quad i=1,2, \ldots, n_{u}$ satisfying (36) such that for some $\delta_{1}>0$

$$
\begin{aligned}
& (X-W(j \omega))\left(M_{11}(j \omega)+I\right)+\left(M_{11}^{*}(j \omega)+I\right)\left(X-W^{*}(j \omega)\right) \geq \delta_{1} I, \forall \omega \in \Re, \\
& \text { where, } W(j \omega)=\operatorname{diag}\left(W_{1}(j \omega), \ldots, W_{n_{u}}(j \omega)\right), X=\operatorname{diag}\left(X_{1}, \ldots, X_{n_{u}}\right)>0 .
\end{aligned}
$$

If $N$ is not odd, then in the stability conditions stated above, we require, in addition, $w_{i}$ to satisfy

$$
w_{i}(t) \geq 0, \forall t \in(-\infty, \infty) .
$$

Proof. The proof involves application of Corollary 1 with the multiplier $X-W(s)$ and is given in Appendix A. It requires several intermediate results which are extensions of the scalar results from [33] to the multivariable case.

Theorem 4 generalizes the SISO AWBT stability result in [34] which was obtained by applying the Off-axis Circle Criterion [11]. In fact, the multiplier in the SISO Off-axis Circle Criterion is of the form $e^{j \theta}, \theta \in\left(-\frac{\pi}{2}, \frac{\pi}{2}\right)$ which can be obtained as a limiting case of the elements of the class of SISO RC and RL multipliers [31], as the number of terms in the $\mathrm{RC} / \mathrm{RL}$ multipliers tends to infinity. Note that the RC and RL multipliers introduced in [31] are special cases of the multipliers characterized in Theorem 4 by equation (36).

The AWBT stability conditions in Theorem 4 are not very useful since they are not constructive, i.e., it is not clear how to search for the infinite dimensional, non-causal operators $w_{i}(t), i=1, \ldots, n_{u}$, satisfying (36), (37), (38) and (39). One alternative is to decompose $w_{i}(t)$ into causal and anti-causal components and then approximate each component by a finite dimensional LTI system. Such an approach and a complete solution involving LMIs has been presented in [10]. For completeness, we briefly discuss this approach here. Details can be found in [10].

Let us express $w_{i}(t)$ in terms of its causal and anti-causal components as $w_{i}(t)=w_{i}^{+}(t)+$ $w_{i}^{-}(t)$, where

$$
w_{i}^{+}(t)=\left\{\begin{array}{ll}
w_{i}(t) & \text { if } t \geq 0, \\
0 & \text { if } t<0 ;
\end{array} \quad w_{i}^{-}(t)= \begin{cases}w_{i}(t) & \text { if } t \leq 0 \\
0 & \text { if } t>0 .\end{cases}\right.
$$

We can now obtain finite series expansions of $w_{i}^{+}(t)$ and $w_{i}^{-}(t)$ with basis functions $e_{j}^{+}(t)=$ $e^{-t} t^{j}, t \geq 0$, (zero for $t<0$ ) and $e_{j}^{-}(t)=e^{t} t^{j}, t \leq 0$, (zero for $t>0$ ) respectively. This leads to an $m^{\text {th }}$ order approximation of $w_{i}(t)$ as follows:

$$
w_{i}(t)=\sum_{j=0}^{m}\left(a_{i, j} e_{j}^{+}(t)+b_{i, j} e_{j}^{-}(t)\right) .
$$


This is equivalent to using $\frac{1}{(s+1)^{j}}$ and $\frac{1}{(s-1)^{j}}, j \geq 1$, as basis functions for approximating the causal and anti-causal components of $W_{i}(s)$ respectively. Condition (39), i.e., $w_{i}(t) \geq 0, t \in$ $(-\infty, \infty)$ can be shown to be equivalent to (see [10])

$$
\frac{\sum_{j=0}^{m} a_{i, j}(-1)^{j} s^{2 j}}{(-s+1)^{m}(s+1)^{m}} \geq 0 \text { and } \frac{\sum_{j=0}^{m} b_{i, j} s^{2 j}}{(-s+1)^{m}(s+1)^{m}} \geq 0, \forall s=j \omega, \omega \in \Re,
$$

and the condition (36) can be expressed as

$$
\sum_{j=0}^{m}\left(a_{i, j}+(-1)^{j} b_{i, j}\right) j !<X_{i}
$$

By Lemma 1, condition (40) reduces to checking that the state-space matrices of

$$
\frac{\sum_{j=0}^{m} a_{i, j}(-1)^{j} s^{2 j}}{(-s+1)^{m}(s+1)^{m}}=\left[\begin{array}{c|c}
A_{j}^{(a)} & B_{j}^{(a)} \\
\hline C_{j}^{(a)} & D_{j}^{(a)}
\end{array}\right] \text { and } \frac{\sum_{j=0}^{m} b_{i, j} s^{2 j}}{(-s+1)^{m}(s+1)^{m}}=\left[\begin{array}{c|c}
A_{j}^{(b)} & B_{j}^{(b)} \\
\hline C_{j}^{(b)} & D_{j}^{(b)}
\end{array}\right]
$$

satisfy (12). Here, $C_{j}^{(a)}, D_{j}^{(a)}$ are affine in $a_{i, j}$ and $C_{j}^{(b)}, D_{j}^{(b)}$ are affine in $b_{i, j}$ (see [10]). Application of (12) leads to two matrix inequalities which are affine in $C_{j}^{(a)}, D_{j}^{(a)}$ and $C_{j}^{(b)}, D_{j}^{(b)}$, respectively, and hence are LMIs in $a_{i, j}, b_{i, j}$. Condition (41) is an obvious LMI in $a_{i, j}, b_{i, j}$, $X_{i}$.

For absolute stability, Theorem 4 requires that $W(j \omega), M_{11}(j \omega)$ should satisfy $(37)$, which is equivalent, by Lemma 1 , to checking that the state-space matrices of $(X-W(s))\left(M_{11}(s)+\right.$ $I)=\left[\begin{array}{c|c}\tilde{A} & \tilde{B} \\ \hline \tilde{C} & \tilde{D}\end{array}\right]$ satisfy (12). Here, $\tilde{C}$ is affine in $a_{i, j}, b_{i, j}$ and $\tilde{D}$ is affine in $X_{i}$ (see [10]). Hence the matrix inequality resulting from (12) is affine in $a_{i, j}, b_{i, j}, X_{i}$.

If we do not require $w_{i}(t) \geq 0, t \in(-\infty, \infty)$, which is the case when the nonlinearity $N$ is odd, then the above procedure is a bit more involved and we refer the reader to [10] for details.

Thus, an intractable problem of finding an infinite dimensional multiplier satisfying the conditions in Theorem 4 is approximated by a tractable problem of finding a finite dimensional multiplier via the feasibility of a set of convex LMI conditions. It is worth mentioning that this finite dimensional solution approximates the solution to the original problem to an arbitrary accuracy, as the order $m$ of the approximation of $w_{i}(t)$ tends to infinity.

\subsection{Necessary Conditions for AWBT Stability}

Since we are concerned with stability conditions for all $N \in \operatorname{sector}[0, I]$, we immediately get the following necessary condition.

Theorem 5 The AWBT system in Figure 2 is $\mathcal{L}_{2}$ stable for all $N \in \mathcal{N}_{T V}$ (or $\mathcal{N}_{T I}$ ) only if the $A W B T$ controller $\hat{K}(s)$ stabilizes $\hat{P}(s)\left[\begin{array}{cc}I & 0 \\ 0 & N\end{array}\right]$ for all constant gain matrices $N=$ $\operatorname{diag}\left\{N_{1}, N_{2}, \ldots, N_{n_{u}}\right\} \in \Re^{n_{u} \times n_{u}}$ such that $0 \leq N \leq I$. 
Furthermore, if $\hat{P}(s)=\left[\begin{array}{c|cc}\tilde{A} & \tilde{B}_{1} & \tilde{B}_{2} \\ \hline \tilde{C}_{1} & \tilde{D}_{11} & \tilde{D}_{12} \\ \tilde{C}_{2} & \tilde{D}_{21} & \tilde{D}_{22} \\ \tilde{C}_{3} & \tilde{D}_{31} & \tilde{D}_{32}\end{array}\right]$ (actually, $\tilde{D}_{22}=0$ since $P_{22}(\infty)=0$, see $\S 3$ ), then the above statement is equivalent to the existence of a symmetric matrix $Q=Q^{T}>0$ such that the following LMI is satisfied for all constant gain matrices $N=\operatorname{diag}\left\{N_{1}, N_{2}, \ldots, N_{n_{u}}\right\} \in$ $\Re^{n_{u} \times n_{u}}, 0 \leq N \leq I$ :

$$
\begin{gathered}
\mathcal{A}^{T} Q+Q \mathcal{A}<0 \\
\text { where } \mathcal{A}=\left[\begin{array}{cc}
\tilde{A}+\tilde{B}_{2} N T^{-1}\left(H_{2} D \tilde{C}_{2}+\left(I-H_{2}\right) \tilde{C}_{3}\right) & \tilde{B}_{2} N T^{-1} H_{2} C \\
\left(B-H_{1} D\right) \tilde{C}_{2}+H_{1} \tilde{C}_{3}+ & A-H_{1} C+ \\
H_{1} \tilde{D}_{32} N T^{-1}\left(H_{2} D \tilde{C}_{2}+\left(I-H_{2}\right) \tilde{C}_{3}\right) & H_{1} \tilde{D}_{32} N T^{-1} H_{2} C
\end{array}\right]
\end{gathered}
$$

with $T=I-\left(I-H_{2}\right) \tilde{D}_{32} N$.

Proof. Follows trivially by forming the closed-loop $\mathcal{A}$ matrix of $\hat{P}\left[\begin{array}{cc}I & 0 \\ 0 & N\end{array}\right]$ and $\hat{K}$ and using Lyapunov's theorem. Note that the inverse of $T=I-\left(I-H_{2}\right) \tilde{D}_{32} N$ is well-defined for all $0 \leq N \leq I$ since, by assumption (see $\S 3$ ), the loop formed by $N$ is well-posed.

Remark 3 For a SISO nonlinearity $\left(n_{u}=1\right)$, a similar condition was claimed to be sufficient for stability and is the well-known Aizermann's conjecture. That conjecture has since been proved false [17].

By considering the cases $N \equiv 0$ and $N \equiv I$, we get the following corollary from Theorem 5 .

Corollary 2 The AWBT system in Figure 2 is $\mathcal{L}_{2}$ stable for all $N \in \mathcal{N}_{T V}$ (or $\mathcal{N}_{T I}$ ) only if

1. $\hat{P}(s)$ and $\hat{K}(s)$ are stable; and

2. $\hat{K}(s)$ stabilizes $\hat{P}(s)$.

\section{Example}

The plant we consider here is a fourth order lead-lag butterworth filter taken from Doyle et al. (1987) [15]:

$$
P(s)=0.2\left(\frac{s^{2}+2 \xi_{1} \omega_{1} s+\omega_{1}^{2}}{s^{2}+2 \xi_{1} \omega_{2} s+\omega_{2}^{2}}\right)\left(\frac{s^{2}+2 \xi_{2} \omega_{1} s+\omega_{1}^{2}}{s^{2}+2 \xi_{2} \omega_{2} s+\omega_{2}^{2}}\right)
$$

where $\omega_{1}=0.2115, \omega_{2}=0.0473, \xi_{1}=0.3827$ and $\xi_{2}=0.9239$. The control input $u$ to the plant is constrained to lie in the range $[-0.5,0.5]$, i.e., (see Figure 9)

$$
\hat{u}=\operatorname{sat}(u)=\left\{\begin{aligned}
-0.5 & \text { if } u<-0.5 \\
u & \text { if }-0.5 \leq u \leq 0.5 \\
0.5 & \text { if } u>0.5
\end{aligned}\right.
$$




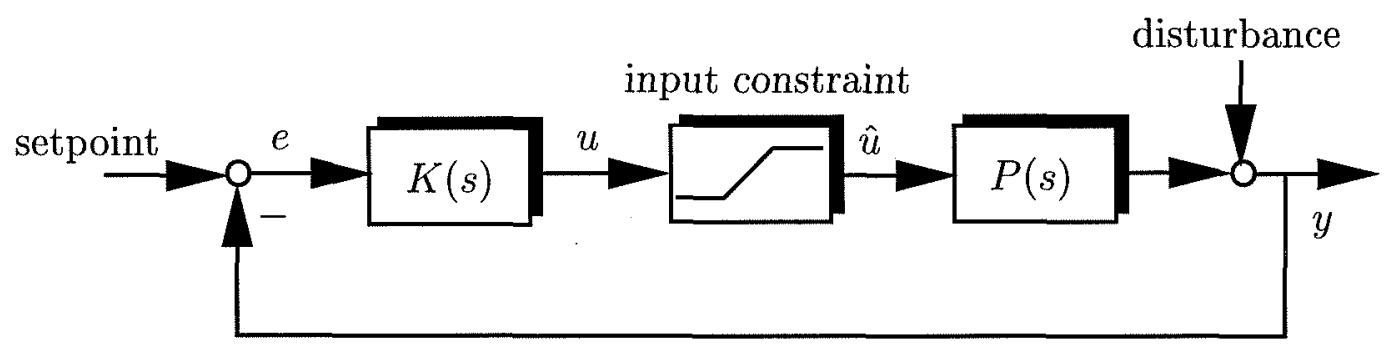

Figure 9: Standard feedback interconnection for the example

In the absence of any input constraints, a PI controller which stabilizes the plant is given by

$$
K(s)=k\left(1+\frac{1}{\tau_{I} s}\right), \text { with } k=100, \tau_{I}=10 .
$$

The feedback interconnection in Figure 9 can be redrawn in the standard form of Figure 1(b) with $N$ in Figure 1(b) corresponding to the saturation nonlinearity in Figure 9.

We would like to analyze the stability properties of typical anti-windup schemes applied to this problem. Several anti-windup schemes and the anti-windup controller $\hat{K}(s)$ corresponding to Figure 2 are listed below (see [24] for a description of these techniques). The corresponding values of the matrix parameters $H_{1}$ and $H_{2}$.in the general AWBT framework of $\S 2.1$, as summarized in Table 1 , are also listed.

- Classical anti-reset windup:

$$
\hat{K}(s)=\left[\begin{array}{c|cc}
-\frac{1}{\tau_{r}} & \frac{k}{\tau_{I} \tau_{r}}\left(\tau_{r}-\tau_{I}\right) & \frac{1}{\tau_{r}} \\
\hline 1 & k & 0
\end{array}\right], H_{1}=\frac{1}{\tau_{r}}, H_{2}=1,
$$

where $\tau_{r}$ is the so-called reset time constant.

- Hanus' conditioned controller:

$$
\hat{K}(s)=\left[\begin{array}{c|cc}
-\frac{1}{\tau_{I}} & 0 & \frac{1}{\tau_{I}} \\
\hline 1 & k & 0
\end{array}\right], H_{1}=\frac{1}{\tau_{I}}, H_{2}=1 .
$$

- Generalized conditioned controller:

$$
\hat{K}(s)=\left[\begin{array}{c|cc}
-\frac{k}{\tau_{I}(k+\rho)} & \frac{k \rho}{\tau_{I}(k+\rho)} & \frac{k}{\tau_{I}(k+\rho)} \\
\hline 1 & k & 0
\end{array}\right], H_{1}=\frac{k}{\tau_{I}(k+\rho)}, H_{2}=1,
$$

where, $\rho$ is a tuning parameter.

- Observer-based anti-windup:

$$
\hat{K}(s)=\left[\begin{array}{c|cc}
-L & \frac{k}{\tau_{I}}-L k & L \\
\hline 1 & k & 0
\end{array}\right], H_{1}=L, H_{2}=1,
$$

where, $L$ is the observer gain. 
AWBT design for these seemingly different techniques can be considered as the single problem of choosing an appropriate $H_{1}$ (or equivalently, $L$ in the observer-based anti-windup scheme) since a given value of $H_{1}$ corresponds to unique values of the AWBT parameters $\tau_{r}, \rho$ and $L$ in these techniques. Note that the Hanus' conditioned controller has no free AWBT parameters to "tune" or optimize nonlinear performance.

Table 2 shows the results of applying the stability tests from Theorems 2, 3, 4 (using a finite series expansion of the multiplier), and the off-axis circle criterion, for various values of $H_{1}=L$ in the observer-based anti-windup scheme. The corresponding multipliers $X-W(s)$

\begin{tabular}{||l|l|l|l|l||}
\hline \hline$H_{1}=L$ & 1 & 10 & 100 & 10000 \\
\hline $\begin{array}{l}\text { Theorem 2 } \\
\text { (Circle Criterion) }\end{array}$ & inconclusive & inconclusive & inconclusive & inconclusive \\
\hline $\begin{array}{l}\text { Theorem 3 } \\
\text { (Popov Criterion) }\end{array}$ & inconclusive & inconclusive & inconclusive & inconclusive \\
\hline Off-axis circle criterion & inconclusive & inconclusive & inconclusive & inconclusive \\
\hline Theorem 4 & stable & stable & stable & stable \\
\hline \hline
\end{tabular}

Table 2: Application of various AWBT stability conditions

establishing stability for the four cases above, using Theorem 4 and the finite dimensional approximation of the multiplier, as discussed in $§ 3.2 .3$, are given respectively by.

- $L=1$ :

$$
1.4118-\frac{2.0237}{s+1}+\frac{0.7659}{(s+1)^{2}}-\frac{0.1534}{(s+1)^{3}}-\frac{6.072 \times 10^{-4}}{s-1}-\frac{5.9974 \times 10^{-4}}{(s-1)^{2}}-\frac{5.04 \times 10^{-4}}{(s-1)^{3}} .
$$

- $L=10$ :

$$
1234.6-\frac{183.442}{s+1}-\frac{1048.8}{(s+1)^{2}}-\frac{0.5927}{s-1}+\frac{0.6012}{(s-1)^{2}}
$$

- $L=100$ :

$$
19.7278-\frac{1.2138}{s+1}-\frac{18.47}{(s+1)^{2}}-\frac{0.0116}{s-1}+\frac{0.0127}{(s-1)^{2}}
$$

- $L=10000$ :

$$
346.59-\frac{18.65}{s+1}-\frac{326.97}{(s+1)^{2}}-\frac{0.2407}{s-1}+\frac{0.2415}{(s-1)^{2}}
$$

A simple Nyquist plot like the one shown in Figure 10(a) can be used to verify that in each case, these multipliers satisfy the frequency domain condition (31) of Theorem 4 . The Nyquist plot of $M_{11}(s)+1$ is shown in Figure 10(b). Comparing the two Nyquist plots, we see that by multiplying $M_{11}(s)+1$ with the multiplier $X-W(s)$, we effectively move the Nyquist plot to the right of the imaginary axis, as required by the stability condition in Theorem 4. 


\section{Conclusions}

In this paper, we presented a general approach for analyzing the stability properties of AWBT control systems. The approach involved application of the passivity theorem with suitable choice of multipliers to develop sufficient conditions for stability. This AWBT stability analysis framework allowed us to consider any multivariable linear AWBT control system subject to multivariable control input nonlinearities. In the same setting, we could deal with several classes of input nonlinearities encountered in operating control systems, such as saturation, relay, dead-zone, hysteresis, switching/override/logic-based nonlinearities and combinations thereof.

The basic premise was to cover the input nonlinearity by a class of sector bounded memoryless structured nonlinearities and then apply concepts from absolute stability theory to develop sufficient conditions guaranteeing stability for all nonlinearities in the specified class. Indeed, this has been the predominant approach to analyzing stability properties of AWBT control system reported in the literature $[9,8,15,19,20,21,22]$. These previous attempts to analyze AWBT stability properties were based on application of seemingly diverse results and theorems to the AWBT problem. Our approach generalizes these previous attempts to analyze AWBT stability. This generalization comes from two sources:

- The AWBT framework from Kothare et al. [24], which is central to the AWBT stability problem under consideration, unifies all known LTI AWBT schemes reported in the literature.

- The multiplier approach to stability analysis used in this paper has been shown to be a generalization of several seemingly diverse stability analysis techniques [4, 5, 28]. Similarly, the connection between the multiplier approach and conventional Lyapunov stability analysis is also well-established [3].

Thus, Theorems 2 and 3 generalize the results from $[2,8,9,15,22]$ and $[19,20,21]$ respectively, which were derived using small-gain arguments, $\mu$ upper bounds, a version of the multiloop Circle Criterion, describing functions and the SISO Popov Criterion. Theorem 4, in its general form, has never been used for analyzing AWBT stability. One particular case which it generalizes is the Off-axis Circle Criterion which was used in [34] for analyzing stability of the anti-windup IMC scheme.

Moreover, our sufficient conditions for AWBT stability, derived under various restrictions on the input nonlinearity, can be checked easily via the feasibility of equivalent convex LMI conditions. In particular, the multiplier establishing stability can be explicitly constructed from the feasible solution to the LMIs. The necessary conditions, derived in $\S 3.3$, give insight into the extent of conservatism involved in the sufficient AWBT stability conditions. Extensions to account for structured plant uncertainty can be worked out in a straightforward manner by augmenting the nonlinear block $N$ with structured, norm-bounded uncertainty blocks and using "mixed" multipliers.

The ultimate goal in studying AWBT control schemes is to develop systematic AWBT synthesis techniques for designing the AWBT matrix parameters $H_{1}$ and $H_{2}$. The analysis results presented in this paper will serve as a starting point in this direction of future research. 


\section{Acknowledgment}

Partial financial support from the U.S. National Science Foundation and the Swiss Federal Institute of Technology (ETH), Zürich, Switzerland, is gratefully acknowledged. We would like to thank Prof. B. D. O. Anderson for several helpful comments.

\section{A Appendix A: Proof of Theorem 4}

We will need several subsidiary lemmas before we can prove Theorem 4. Most of these lemmas are straightforward extensions of the scalar results from [33] to the multivariable case. Hence, we will only outline the proofs without going into details. We begin with a factorization lemma.

Lemma 3 Let $w_{i}(t), i=, 1,2, \ldots, n_{u}$ be the impulse response of a scalar LTI (possibly noncausal) operator on $t \in(-\infty, \infty)$ satisfying (36) for some $X_{i}>0$. Let $W_{i}(s)$ be its Laplace transform

$$
W_{i}(s)=\int_{-\infty}^{\infty} e^{-s t} w_{i}(t) d t, i=1,2, \ldots, n_{u}
$$

Let $W(s)=\operatorname{diag}\left(W_{1}(s), W_{2}(s), \ldots, W_{n_{u}}(s)\right)$ and $X=\operatorname{diag}\left(X_{1}, X_{2}, \ldots, X_{n_{u}}\right)>0 . \quad$ Then, there exist matrix tranfer functions $W_{+}(s)$ and $W_{-}(-s)$ which are stable and proper with stable and proper inverses such that

$$
X-W(s)=W_{-}(s) W_{+}(s) .
$$

Proof. From [33, Lemma 3], it follows that since $\int_{-\infty}^{\infty}\left|w_{i}(t)\right| d t<X_{i}$, there exist scalar transfer functions $W_{i-}(-s), W_{i+}(s)$ which are stable and proper with stable and proper inverses such that

$$
X_{i}-W_{i}(s)=W_{i-}(s) W_{i+}(s), \quad i=1,2, \ldots, n_{u} .
$$

Choosing $W_{-}(s)=\operatorname{diag}\left(W_{1-}(s), \ldots, W_{n_{u}-}(s)\right), W_{+}(s)=\operatorname{diag}\left(W_{1+}(s), \ldots, W_{n_{u}+}(s)\right)$ then establishes the lemma.

Lemma 4 Let $w_{i}(t), W_{i}(s), W(s), X_{i}, X$ be as in Lemma 3. Let $X-W(s)$ be factorized as $W_{-}(s) W_{+}(s)$ as in Lemma 3. Let $N=\operatorname{diag}\left\{N_{1}, \ldots, N_{n_{u}}\right\}$ be a static, monotone nondecreasing, passive nonlinearity. If either $N_{i}$ is odd or $w_{i}(t) \geq 0, t \in \Re$, then $W_{-}(-s)^{T} \circ$ $N \circ W_{+}(s)^{-1}$ is passive.

Proof. From [33, Proposition 1], since $\int_{-\infty}^{\infty}\left|w_{i}(t)\right| d t<X_{i}$ and either $N_{i}$ is odd or $w_{i}(t) \geq$ $0, t \in \Re$, we conclude that $\left(X_{i}-W_{i}(-s)\right) \circ N_{i}$ is passive, i.e.,

$$
\begin{gathered}
\left\langle x_{i T} \mid\left[\left(X_{i}-W_{i}(-s)\right) \circ N_{i} x_{i}\right]_{T}\right\rangle \geq 0, \forall x_{i} \in \Re, T \geq 0 \\
\Leftrightarrow\left\langle x_{i T} \mid\left[W_{i+}(-s) W_{i-}(-s) \circ N_{i} x_{i}\right]_{T}\right\rangle \geq 0, \forall x_{i} \in \Re, T \geq 0 \\
\Leftrightarrow\left\langle\left[W_{i+}(s) x_{i}\right]_{T} \mid\left[W_{i-}(-s) \circ N_{i} x_{i}\right]_{T}\right\rangle \geq 0, \forall x_{i} \in \Re, T \geq 0 \\
\left.\Leftrightarrow\left\langle y_{i T} \mid\left[W_{i-}(-s) \circ N_{i} \circ W_{i+}(s)^{-1} y_{i}\right]_{T}\right\rangle \geq 0, \forall y_{i} \in \Re, T \geq 0 \text { (substituting } y=W_{i+}(s) x_{i}\right) .
\end{gathered}
$$

Hence $W_{i-}(-s)^{T} \circ N_{i} \circ W_{i+}(s)^{-1}$ is passive. Since $W_{-}(-s)^{T} \circ N \circ W_{+}(s)^{-1}$ is a diagonal operator with all its diagonal entries passive, hence it is passive. 
Lemma 5 Let $N=\operatorname{diag}\left\{N_{1}, \ldots, N_{n_{u}}\right\}$ be a diagonal nonlinearity satisfying the conditions in Definitions 4 and 5. Suppose we apply the loop transformation of Figure 6 to $N$ to get a diagonal nonlinearity $\tilde{N}=\operatorname{diag}\left\{\tilde{N}_{1}, \ldots, \tilde{N}_{n_{u}}\right\}$ with $\tilde{N}_{i} \in \operatorname{sector}[0, \infty]$. Then, if $N$ is odd and monotone non-decreasing, so is $\tilde{N}$.

Proof. Since $N=\operatorname{diag}\left\{N_{1}, \ldots, N_{n_{u}}\right\}$, if $N$ is odd, monotone non-decreasing, then so are $N_{i}, i=1,2, \ldots, n_{u}$. From [33, $\left.\S 7\right]$, we conclude that if $N_{i}$ is odd, monotone non-decreasing, then so is $\tilde{N}_{i}$. The lemma then follows since $\tilde{N}=\operatorname{diag}\left\{\tilde{N}_{1}, \ldots, \tilde{N}_{n_{u}}\right\}$.

Proof of Theorem 4. As in the proofs of Theorems 2 and 3, we can show that $M(s)$ is stable and the $M_{11}(s)-N$ loop is well-posed. Also, we can transform the $M_{11}(s)-N$ loop to the $\tilde{M}_{11}(s)-\tilde{N}$ loop where $\tilde{M}_{11}(s)=M_{11}(s)+I$ and $\tilde{N}=\operatorname{diag}\left\{\tilde{N}_{1}, \ldots, \tilde{N}_{n_{u}}\right\}, \tilde{N}_{i} \in$ sector $[0, \infty]$, with $\tilde{N}$ passive. From Lemma 5, we conclude that if $N$ is odd, monotone non-decreasing and incrementally inside sector $[0, I]$, then $\tilde{N}$ is odd and monotone non-decreasing.

We can now apply Corollary 1 to the $\tilde{M}_{11}(s)-\tilde{N}$ loop with $H(s)=\tilde{M}_{11}(s)$ and $f=\tilde{N}$. The appropriate multiplier is $X-W(s)$, where $W(s)=\operatorname{diag}\left(W_{1}(s), \ldots, W_{n_{u}}(s)\right)$, with the impulse responses $w_{i}(t)$ of the scalar transfer functions $W_{i}(s)$ satisfying $(36)$. From Lemma 3 , we conclude that $X-W(s)$ can be factorized into $W_{-}(s) W_{+}(s)$ which satisfy conditions 1 and 2 of Corollary 1 . By Lemma 4, (17) of Corollary 1 holds with $\delta_{2}=0$. (16) and (18) can be checked via (19) (see Remark 2) as follows:

$$
(X-W(j \omega))\left(M_{11}(j \omega)+I\right)+\left(M_{11}^{*}(j \omega)+I\right)\left(X-W^{*}(j \omega)\right) \geq \delta_{1} I, \forall \omega \in \Re
$$

which establishes (37) and the proof is complete.

\section{References}

[1] B. D. O. Anderson and S. Vongpanitlerd. Network Analysis and Synthesis: A Modern Systems Theory Approach. Prentice-Hall, Inc., Englewood Cliffs, N.J., 1973.

[2] K. J. Åström and L. Rundqwist. Integrator windup and how to avoid it. In Proceedings of the 1989 American Control Conference, pages 1693-1698, 1989.

[3] V. Balakrishnan. Construction of Lyapunov functions for robustness analysis with multipliers. In Proceedings of the $33^{\text {rd }}$ IEEE Conference on Decision and Control, pages 2021-2025, Orlando, Florida, December 1994.

[4] V. Balakrishnan. Linear matrix inequalities in robustness analysis with multipliers. Systems \& Control Letters, 25(4):265-272, July 1995.

[5] V. Balakrishnan, Y. Huang, A. Packard, and J. Doyle. Linear matrix inequalities in analysis with multipliers. In Proceedings of the 1994 American Control Conference, pages 1228-1232, Baltimore, Maryland, June 1994.

[6] S. Boyd, L. El Ghaoui, E. Feron, and V. Balakrishnan. Linear Matrix Inequalities in System and Control Theory, volume 15 of Studies in Applied Mathematics. SIAM, Philadelphia, PA, June 1994. 
[7] R. W. Brockett and J. L. Willems. Frequency domain stability criteria-Parts I and II. IEEE Trans. Auto. Cont., 10(3,4):255-261, 407-412, 1965.

[8] P. J. Campo and M. Morari. Robust control of processes subject to saturation nonlinearities. Computers $\&$ Chemical Engineering, 14(4/5):343-358, 1990.

[9] P. J. Campo, M. Morari, and C. N. Nett. Multivariable anti-windup and bumpless transfer: A general theory. In Proceedings of the 1989 American Control Conference, 1989.

[10] X. Chen and J. T. Wen. Robustness analysis of LTI systems with structured incrementally sector bounded nonlinearities. In Proceedings of the 1995 American Control Conference, pages 3883-3887, July 1995.

[11] Y.-S. Cho and K. S. Narendra. An off-axis circle criterion for the stability of feedback systems with a monotonic nonlinearity. IEEE Trans. Auto. Cont., 13:413-416, August 1968.

[12] C. A. Desoer and M. Vidyasagar. Feedback Systems: Input-Output Properties. Academic Press, New York, 1975.

[13] J. C. Doyle, K. Glover, P. Khargonekar, and B. Francis. State-space solutions to standard $\mathcal{H}_{2}$ and $\mathcal{H}_{\infty}$ control problems. IEEE Trans. Auto. Cont., 34(8):831-847, Aug 1989.

[14] J. C. Doyle and A. Packard. Uncertain multivariable systems from a state space perspective. In Proceedings of the 1987 American Control Conference, pages 2147-2152, Minneapolis, MN, 1987.

[15] J. C. Doyle, R. S. Smith, and D. F. Enns. Control of plants with input saturation nonlinearities. In Proceedings of the 1987 American Control Conference, pages 10341039, Minneapolis, MN, 1987.

[16] H. A. Fertik and C. W. Ross. Direct digital control algorithm with anti-windup feature. ISA Transactions, 6(4):317-328, 1967.

[17] R. E. Fitts. Two counterexamples to Aizerman's conjecture. IEEE Trans. Auto. Cont, 11(3):553-556, 1966.

[18] P. Gahinet, A. Nemirovski, A. J. Laub, and M. Chilali. LMI Control Toolbox: For use with MATLAB. The Mathworks, Inc., Natick, MA, May 1995.

[19] A. H. Glattfelder and W. Schaufelberger. Stability analysis of single-loop control systems with saturation and antireset-windup circuits. IEEE Trans. Auto. Cont., 28(12):10741081, December 1983.

[20] A. H. Glattfelder and W. Schaufelberger. Stability of discrete override and cascadelimiter single-loop control systems. IEEE Trans. Auto. Cont., 33(6):532-540, June 1988. 
[21] A. H. Glattfelder, W. Schaufelberger, and H. P. Fassler. Stability of override control systems. Int. J. Control, 37(5):1023-1037, 1983.

[22] P. Kapasouris and M. Athans. Multivariable control systems with saturating actuators, antireset windup strategies. In Proceedings of the 1985 American Control Conference, pages $1579-1584,1985$.

[23] H. K. Khalil. Nonlinear Systems. Prentice Hall, Inc., Upper Saddle River, NJ, second edition, 1996.

[24] M. V. Kothare, P. J. Campo, M. Morari, and C. N. Nett. A unified framework for the study of anti-windup designs. Automatica, 30(12):1869-1883, 1994. Also presented at the 1993 AIChE Annual Meeting, MO.

[25] A. I. Lur'e. Some nonlinear problems in the theory of automatic control. Her Majesty's Stationery Office, London, 1957. In Russian, 1951.

[26] A. Packard and J. Doyle. The complex structured singular value. Automatica, 29(1):71109, 1993.

[27] V. M. Popov. Absolute stability of nonlinear systems of automatic control. Automatic Remote Control, 22(8):857-875, 1961.

[28] M. G. Safonov and G. Wyetzner. Computer-aided stability analysis renders Popov criterion obsolete. IEEE Trans. Auto. Cont., 32(12):1128-1131, December 1987.

[29] I. W. Sandberg. On the $\mathcal{L}_{2}$-boundedness of solutions of nonlinear functional equations. Bell Syst. Tech. J., 43(4):1581-1589, 1964.

[30] G. Zames. On the input-output stability of time varying nonlinear feedback systems Part I: Conditions derived using concepts of loop gain, conicity, and positivity. IEEE Trans. Auto. Cont., 11(2):228-238, April 1966.

[31] G. Zames. On the input-output stability of time varying nonlinear feedback systems - Part II: Conditions involving circles in the frequency plane and sector nonlinearities. IEEE Trans. Auto. Cont., 11(3):465-476, July 1966.

[32] G. Zames. Stability of systems with sector nonlinearities: A comparison of various inequalities. IEEE Trans. Auto. Cont., 13(6):709-711, December 1968.

[33] G. Zames and P. L. Falb. Stability conditions for systems with monotone and sloperestricted nonlinearities. SIAM J., 6(1):89-108, 1968.

[34] A. Zheng, M. V. Kothare, and M. Morari. Anti-windup design for internal model control. International Journal of Control, 60(5):1015-1024, 1994. Also presented at the 1993 AIChE Annual Meeting, MO. 
(a) $(X-W(s))\left(M_{11}(s)+1\right)$

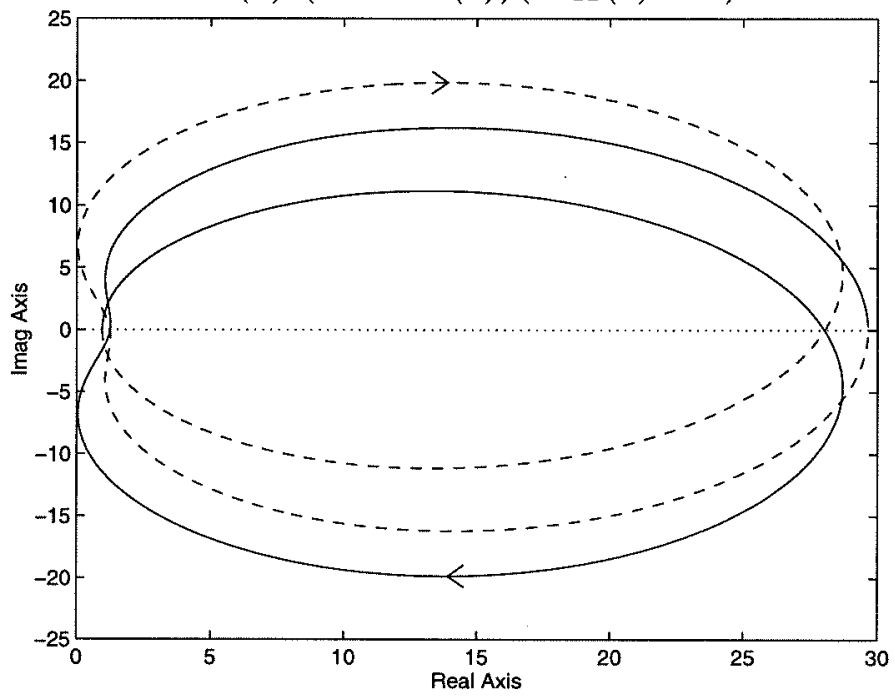

(b) $M_{11}(s)+1$

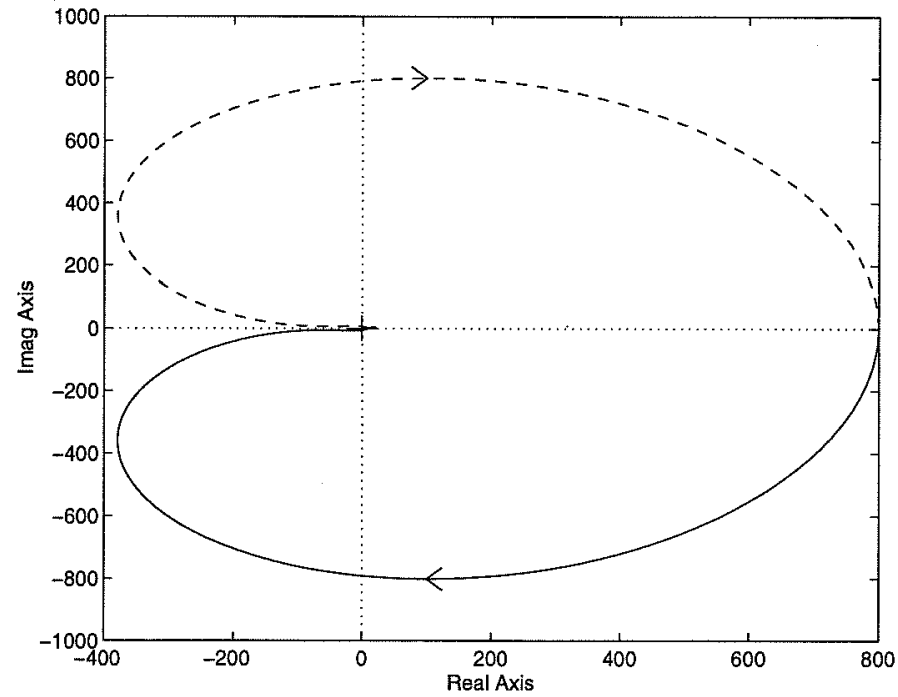

Figure 10: Nyquist plot of (a) $(X-W(s))\left(M_{11}(s)+1\right)$, and (b) $M_{11}(s)+1$ for the case $L=1$ 